\title{
Reconsidering XPS Quantification of Substitution Levels of Monolayers on Unoxidized Silicon Surfaces
}
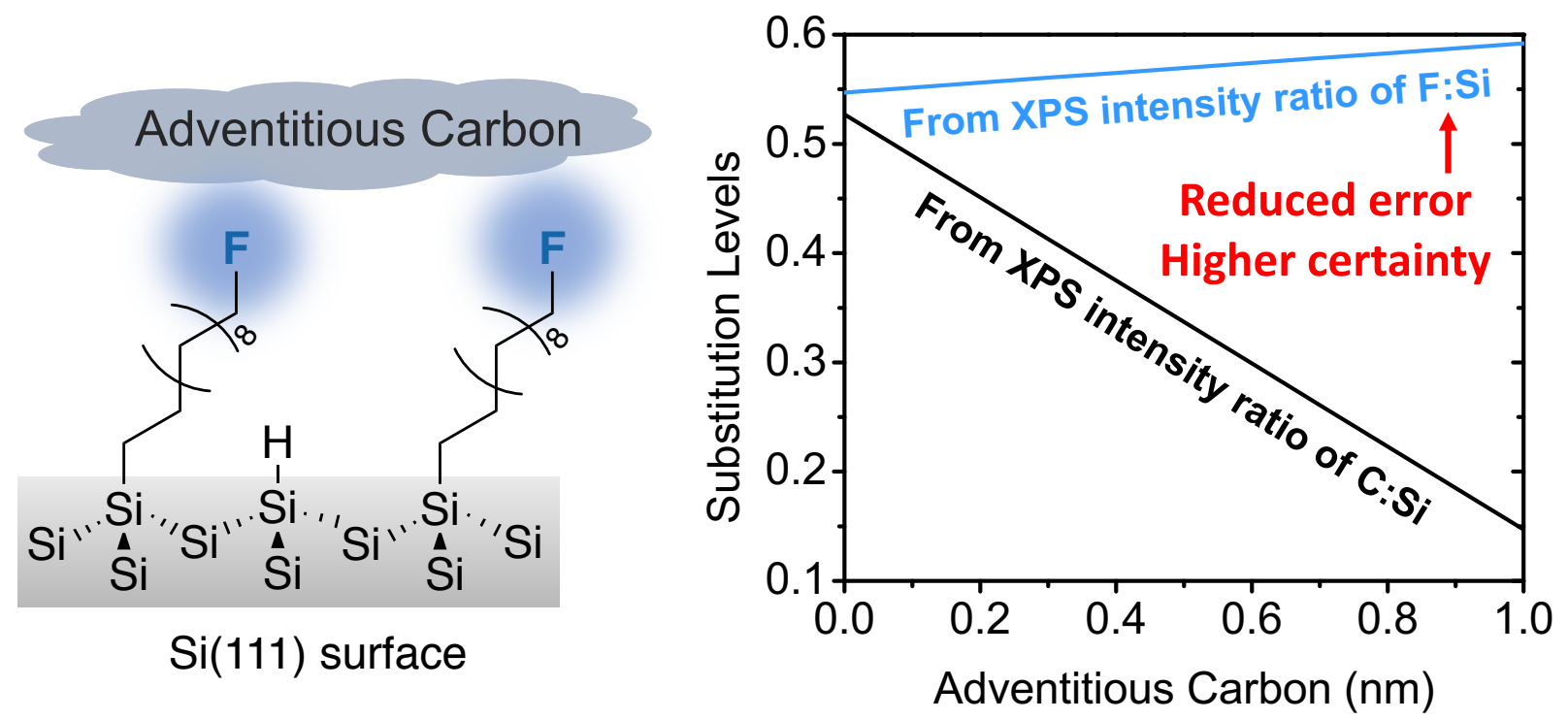

Minjia Hu, Erik J. Luber,* Jillian M. Buriak*

Department of Chemistry, University of Alberta, 11227 Saskatchewan Drive, Edmonton, Alberta T6G 2G2, Canada

NRC-Nano, National Research Council Canada, 11421 Saskatchewan Drive, Edmonton, Alberta T6G 2M9, Canada 


\begin{abstract}
The covalent functionalization of unoxidized silicon surfaces is of interest for a wide range of applications, and for fundamental studies linking surface functionalization and electronic properties. Determination of the level of substitution (yield) of a reaction on a silicon surface is necessary as the number of functional groups bound to the surface is directly linked to properties. X-ray photoelectron spectroscopy (XPS), is the most common analytical method for determining the substitution level of the chemical handle on the silicon surface, typically a $\mathrm{Si}-\mathrm{H}$ or $\mathrm{Si}-\mathrm{Cl}$ bond, through which a new stable bond is formed to link the molecule to the surface. Calculations using the atomic ratio of carbon to silicon as determined by XPS do not take into account the effect of adventitious carbon, retained solvent and the substitution level is typically measured by first assuming 100\% substitution of a fictitious hydrocarbon layer with an effective thickness that is determined by XPS intensity ratio of $\mathrm{C}$ to $\mathrm{Si}$, and then the real substitution level is taken as the ratio of the effective thickness to the theoretical height of the molecule. In this work, we take an alternative and more physically meaningful approach to deriving expressions for the substitution level, where the photoelectron attenuation length is proportional to the substitution level. For all-hydrocarbon molecules grafted to a silicon surface, this new approach yields the same equations for substitution levels as an earlier effective thickness model. More importantly, unlike the effective thickness models, this method can be extended to include molecules with a heteroatom "tag", such as fluorine and chalcogenides, for determining coverage by XPS; this latter approach is shown to provide a greater degree of certainty with respect to calculating coverage on silicon. We finish with a simple flowchart to guide the reader to the appropriate equation for both $\mathrm{Si}(111)$ and $\mathrm{Si}(100)$ surfaces.
\end{abstract}




\section{- Introduction}

Modification of unoxidized silicon surfaces via the grafting of organic molecules through direct covalent bonds to the silicon is of great interest to tailor the physical and chemical properties of the underlying semiconductor materials. ${ }^{1-3}$ Many routes to functionalizing silicon surfaces through $\mathrm{Si}-\mathrm{C}, \mathrm{Si}-\mathrm{E}$ (E = chalcogenide), and $\mathrm{Si}-\mathrm{N}$ bonds have been developed, with the goal of controlling interfacial electronic properties, ${ }^{4-22}$ passivating the surface, ${ }^{23,24}$ and enabling the linking of a diverse array of sensor elements, ${ }^{25-32}$ among others. These studies and applications would require knowledge of surface coverage of the functional groups bound to the surface as variable substitution levels would result in a lowered degree of reproducibility.

Experimentally, determination of percent substitution of available silicon atoms on the surface of atomically flat, terraced $\mathrm{Si}(111)$ surfaces has relied upon a variety of methods, including X-ray photoelectron spectroscopy (XPS), ellipsometry, scanning tunneling microscopy (STM), Fourier transform infrared (FTIR) spectroscopy, and X-ray reflectivity, often in concert with each other. ${ }^{33-43}$ In Linford and Chidsey's landmark work of 1993 and 1995 that introduced alkene hydrosilylation on $\mathrm{Si}(111)-\mathrm{H}$, they hypothesized that maximum surface coverage was reached when $\sim 50 \%$ of surface silicon-hydride groups were substituted with alkyl groups. ${ }^{44,45}$ Soon after, Südholter and Zuilhof applied molecular modeling simulations to obtain more detailed information about the structure and average substitution values of alkyl monolayers on silicon surfaces. Using two-dimensionally repeating boxes to mimic the hydrosilylated surface, the effects of various substitution levels $(33.3 \%, 50 \%, 66.7 \%$, and $100 \%)$ were examined. ${ }^{46} \mathrm{~A}$ substitution level of $\sim 50 \%$ for unbranched alkyl chains provided good agreement with available experimental data and represents a balance of volume of the alkyl groups (calculated using van der Waals radii), the attractive van der Waals forces between the alkyl chains, and experimentally determined average tilt of the alkyl chains within the monolayer. This work was followed up by molecular mechanics simulations by Zhang, ${ }^{47}$ and Yuan, ${ }^{48}$ whose results support the claim that $\sim 50 \%$ substitution is the maximum that can be obtained with long-chain alkyl or alkenyl groups on H-terminated $\mathrm{Si}(111)$.

Shown in Table 1 is a summary of the percent substitution of levels on $\mathrm{Si}(111)$ surfaces, reported in the literature. The data in this table includes both experimental and molecular modeling 
results. For linear alkyl and alkenyl chains longer than 3 carbon units, both experiments and molecular modeling seem to converge upon 40-60\% substitution, although there remains some controversy as to whether coverage depends upon aliphatic chain length. ${ }^{36}$ Complete $100 \%$ coverage of the $\mathrm{Si}(111)$ surface has only been observed when the organic substituent is very small, compact, and highly ordered, such as methyl, acetylenyl or methylacetylene groups. ${ }^{38,49}$ With a van der Waals radius of only $2.2 \AA$, the methyl group has the correct sterics to occupy every apical silicon atom on the $\operatorname{Si}(111)$ surface, which are separated by a distance of $3.8 \AA .^{50}$ With an ethyl-terminated $\mathrm{Si}(111)$ surface, $\sim 80 \%$ of the $\mathrm{Si}$ atop atoms were determined to be substituted by $\mathrm{Si}-\mathrm{C}$ bonds, ${ }^{39}$ and compact propenyl chains on $\mathrm{Si}(111), \equiv \mathrm{Si}-\mathrm{CH}=\mathrm{CH}-\mathrm{CH}_{3}$, were recently reported to have $92 \%$ surface coverage. ${ }^{42}$

As can be seen from Table 1, XPS is the most commonly used method to determine substitution levels on silicon surfaces. Some questions have been raised, however, as to the reliability of XPS for making such determinations. In 2017, Lewis and co-workers noted that values for coverage of methoxy groups on Si(111), as determined by XPS and FTIR differed substantially; XPS yielded values as high as 50\%, whereas FTIR was $\sim 30 \% .{ }^{51}$ They attributed the difference to adventitious adsorption of residual solvent (methanol and/or THF), which would lead to an overestimation of the carbon:silicon ratio and hence result in an inaccurate value of surface coverage. Adventitious hydrocarbon contamination (often referred to as adventitious carbon) has also been noted by Yamazaki to be a potential problem in XPS measurements of surface coverage of hydrocarbon-based monolayers on silicon if one relies solely upon the ratio of carbon C 1s features versus the Si 2p. ${ }^{35}$ This group examined hydrosilylation of alkynes on $\mathrm{Si}(111)-\mathrm{H}$ containing fluorine- and nitrogen-containing groups that were used as atomic labels to quantify coverage using the ratios of $\mathrm{F} 1 \mathrm{~s}$ and $\mathrm{N} 1 \mathrm{~s}$ to $\mathrm{Si} 2 \mathrm{p}$ for their calculations. Our group also noted a discrepancy when using the ratio of $\mathrm{C}$ :Si in XPS to determine the coverage of an octadecanethiol linkage, $\equiv \mathrm{Si}-\mathrm{S}-\left(\mathrm{CH}_{2}\right)_{17}-\mathrm{CH}_{3}$, on $\mathrm{Si}(111)-\mathrm{H}$ : calculations using the silicon:carbon ratio indicated that $\sim 50 \%$ of the $\equiv \mathrm{Si}-\mathrm{H}$ groups were replaced with $\equiv \mathrm{Si}-\mathrm{S}-\left(\mathrm{CH}_{2}\right)_{17}-$ $\mathrm{CH}_{3}$, whereas using the $\mathrm{Si}: \mathrm{S}$ ratio resulted in a substantially lower value of $27 \% .{ }^{43}$ Adjusting the value by subtracting the experimentally determined value for the thickness of adventitious hydrocarbon contamination gettered on hydrophobic $\mathrm{Si}(111)-\mathrm{H}$, however, led to a similar value of $27 \%$ substitution. 
In this work, we reexamine the calculations and presumptions underlying the use of XPS for determining the percent substitution of $\mathrm{Si}(111)-\mathrm{H}$ with alkyl functionalities. Adventitious carboncontaining contamination is ubiquitous as it arises from exposure of a surface to a variety of conditions, including laboratory ambient, ${ }^{52-54}$ wet chemical treatment, ${ }^{51,55}$ and even high vacuum conditions ${ }^{56}$ including within the XPS instrument itself. Contamination may also arise from the physisorption of the solvents and reagents that are not removed during the final washing steps upon completion of the reaction. The potential effect of adventitious and/or physisorbed hydrocarbons on these XPS data-based calculations is considered here in a quantitative manner and contrasted with the determination of substitution levels with monolayers that contain an atomic tag or label (such as F, or chalcogenide); the entire process is then summarized with a simple flowchart for users to follow for their own calculations.

Table 1. Substitution Levels of Alkyl, Alkenyl, or Alkyne Functionalities on Silicon(111) Surfaces Determined by Molecular Modeling and Experiments

\begin{tabular}{|c|c|c|c|c|}
\hline $\begin{array}{l}\text { Functionality, and } \\
\text { silicon surface }^{\mathrm{a}}\end{array}$ & Reaction conditions & $\begin{array}{l}\text { Reported or } \\
\text { hypothesized } \\
\text { substitution } \\
\text { level }\end{array}$ & $\begin{array}{l}\text { Method of determining } \\
\text { substitution level }\end{array}$ & Reference \\
\hline$\equiv \mathrm{Si}(111)-\mathrm{C}_{18}$ & $\begin{array}{l}\text { Alkene with heat or } \\
\text { peroxide Initiator }\end{array}$ & $50 \%$ & $\begin{array}{l}\text { Model based on X-ray } \\
\text { reflectivity, FTIR }\end{array}$ & 45 \\
\hline$\equiv \mathrm{Si}(111)-\mathrm{C}_{8}$ & Alkene with UV light, $2 \mathrm{~h}$ & $44 \%$ & XPS (Si:C ratio) & 33 \\
\hline$=\mathrm{Si}(111)-\mathrm{C}_{18}$ & Alkene with UV light, $2 \mathrm{~h}$ & $45 \%$ & XPS (Si:C ratio) & 33 \\
\hline $\begin{array}{l}=\mathrm{Si}(111)-\mathrm{C}_{n} \\
(n=8,10,12,16 \text {, and } \\
18)\end{array}$ & $\begin{array}{l}\text { Alkene with } 10 \% \text { of } \\
\mathrm{C}_{2} \mathrm{H}_{5} \mathrm{AlCl}_{2} \text { as catalyst }\end{array}$ & $43-55 \%$ & XPS (Si:C ratio) & 34 \\
\hline$\equiv \mathrm{Si}(111)-\mathrm{O}-\mathrm{C}_{10}$ & $\begin{array}{l}\text { Alkene with } 10 \% \text { of } \\
\mathrm{C}_{2} \mathrm{H}_{5} \mathrm{AlCl}_{2} \text { as catalyst }\end{array}$ & $42-52 \%$ & XPS (Si:C ratio) & 34 \\
\hline $\begin{array}{l}\equiv \mathrm{Si}(111)-\mathrm{C}=\mathrm{C}-\mathrm{X} \\
\left(\mathrm{X}=\mathrm{CO}_{2} \mathrm{CH}_{3} \mathrm{CF}_{3},\right. \\
\mathrm{CO}_{2} \mathrm{CH}_{2} \mathrm{CH}_{2} \mathrm{CN}, \\
\mathrm{CO}_{2} \mathrm{CH}_{2} \mathrm{C}_{6} \mathrm{H}_{4}-p- \\
\mathrm{CF}_{3}, \mathrm{CN}, \mathrm{C}_{6} \mathrm{H}_{4}-p- \\
\left.\mathrm{CF}_{3}, \text { or } \mathrm{C}_{6} \mathrm{H}_{4}-p-\mathrm{F}\right)\end{array}$ & $\begin{array}{l}\text { Alkyne at room } \\
\text { temperature, } 24-40 \mathrm{~h}\end{array}$ & $31-56 \%$ & XPS (Si:C, Si:F ratios) & 35 \\
\hline $\begin{array}{l}\equiv \mathrm{Si}(111)-\mathrm{Ph}-\mathrm{R} \\
(\mathrm{R}=m \text {-substitution, } \\
\text { and fluorine tag) }\end{array}$ & $\begin{array}{l}\text { Chlorination of surface, } \\
\text { followed by Grignard or } \\
\text { RLi agents }\end{array}$ & $\begin{array}{l}8 \pm 4 \%- \\
57 \pm 31 \%\end{array}$ & XPS (Si:F) & 57 \\
\hline$\equiv \mathrm{Si}(111)-\mathrm{C}=\mathrm{C}-\mathrm{C}_{n}$ & Alkyne at $100^{\circ} \mathrm{C}$, reduced & $55-6$ & XPS (Si:C ratio) & 36 \\
\hline
\end{tabular}




\begin{tabular}{|c|c|c|c|c|}
\hline $\begin{array}{l}(n=10,12,14 \text {, and } \\
16)\end{array}$ & pressure, $6 \mathrm{~h}$ & $5 \%$ & & \\
\hline $\begin{array}{l}\equiv \mathrm{Si}(111)-\mathrm{C}=\mathrm{C}- \\
\mathrm{C}=\mathrm{C}-\mathrm{C}_{12}\end{array}$ & $\begin{array}{l}\text { Alkyne at } 80^{\circ} \mathrm{C}, \\
\text { overnight }\end{array}$ & $63 \%$ & XPS (Si:C ratio) & 37 \\
\hline$\equiv \mathrm{Si}(111)-\mathrm{CH}_{3}$ & $\begin{array}{l}\text { Chlorination of surface, } \\
\text { followed by } \mathrm{CH}_{3} \mathrm{MgCl}\end{array}$ & $100 \%$ & STM & 38 \\
\hline$\equiv \mathrm{Si}(111)-\mathrm{C}_{2}$ & $\begin{array}{l}\text { Chlorination of surface, } \\
\text { followed by } \mathrm{C}_{2} \mathrm{H}_{5} \mathrm{MgCl}\end{array}$ & $80 \%$ & STM & 39 \\
\hline $\begin{array}{l}\equiv \mathrm{Si}(111)-\mathrm{C} \equiv \mathrm{C}-\mathrm{R} \\
\left(\mathrm{R}=\mathrm{H} \text { or } \mathrm{CH}_{3}\right)\end{array}$ & $\begin{array}{l}\text { Chlorination of surface, } \\
\text { followed by } \mathrm{NaC} \equiv \mathrm{C}-\mathrm{R}(\mathrm{R} \\
\left.=\mathrm{H} \text { or } \mathrm{CH}_{3}\right)\end{array}$ & $100 \%$ & FTIR & 40 \\
\hline $\begin{array}{l}\equiv \mathrm{Si}(111)-\mathrm{CH}=\mathrm{CH}- \\
\left(\mathrm{CH}_{2}\right)_{n}-\mathrm{F} \\
(\mathrm{n}=8,10,12,14 \text {, and } \\
16)\end{array}$ & $\begin{array}{l}\text { Alkyne at } 80^{\circ} \mathrm{C}, \\
\text { overnight }\end{array}$ & $51-58 \%$ & XPS (Si:C, Si:F ratios) & 41 \\
\hline $\begin{array}{l}\equiv \mathrm{Si}(111)-\mathrm{CH}=\mathrm{CH}- \\
\mathrm{CH}_{3}\end{array}$ & Alkyne at $130^{\circ} \mathrm{C}, 16 \mathrm{~h}$ & $92 \%$ & XPS (Si:C ratios) & 42 \\
\hline$\equiv \mathrm{Si}(111)-\mathrm{S}-\mathrm{C}_{18}$ & $\begin{array}{l}\text { Alkene and } \mathrm{UV} \text { at } 80^{\circ} \mathrm{C} \text {, } \\
15 \mathrm{~min}\end{array}$ & $27 \%$ & XPS (Si:C \& Si:S ratios) & 43 \\
\hline$\equiv \mathrm{Si}(111)-\mathrm{C}_{18}$ & Computation & $\sim 50 \%$ & Molecular modeling & 58 \\
\hline$\equiv \mathrm{Si}(111)-\mathrm{C}_{18}$ & Computation & $\sim 50 \%$ & Molecular modeling & 46 \\
\hline$\equiv \mathrm{Si}(111)-\mathrm{C}_{18}$ & Computation & $\sim 50 \%$ & Molecular modeling & 47 \\
\hline$\equiv \mathrm{Si}(111)-\mathrm{C}_{8}$ & Computation & $\sim 50 \%$ & Molecular modeling & 48 \\
\hline$\equiv \mathrm{Si}(111)-\mathrm{C}=\mathrm{C}-\mathrm{C}_{6}$ & Computation & $\sim 50 \%$ & Molecular modeling & 48 \\
\hline
\end{tabular}

${ }^{a}$ Linear unbranched alkyl chains are simplified as $-\mathrm{C}_{\mathrm{n}}$.

\section{- Materials and Methods}

Materials. $\operatorname{Si}(111)$ wafers (n-type, phosphorus doped, $1-10 \Omega \cdot \mathrm{cm}, 525 \pm 25 \mu \mathrm{m}$ ) were obtained from Virginia Semiconductor, Inc. 1-Octene (98\%), 1-dodecene (99.0\%), and 1octadecene (95.0\%) were purchased from Sigma-Aldrich. 11-Fluoro-1-undecene (95\%) was obtained from HongKong Chemhere Co.,Ltd. All reagents were stored in a $-20{ }^{\circ} \mathrm{C}$ freezer inside an argon-filled glove box. Dichloromethane was purified by a solvent purification system (Innovative Technology, Inc.) and dried over molecular sieves for $24 \mathrm{~h}$ in a glovebox prior to use. Molecular sieves (type 4A, 1/16 inch pellets, for selective adsorption), $\mathrm{NH}_{4} \mathrm{OH}$ (aqueous, 
$30 \%$ ), and $\mathrm{HCl}$ (aqueous, $37 \%$ ) were purchased from Caledon Laboratories, $\mathrm{Ltd}$. $\mathrm{NH}_{4} \mathrm{~F}$ (aqueous, $40 \%$, semiconductor grade) was purchased from Transene Company, Inc. $\mathrm{H}_{2} \mathrm{O}_{2}$ (aqueous, $30 \%$ ) and 2-propanol (99.5\%) were obtained from Sigma-Aldrich. HF (49\% aqueous, semiconductor grade) was purchased from J. T. Baker. $\mathrm{KAuCl}_{4} \cdot x \mathrm{H}_{2} \mathrm{O}$ was purchased from Strem Chemicals.

Flat Si(111)-H Preparation. Silicon wafers were cut into $1 \mathrm{~cm} \times 1 \mathrm{~cm}$ squares using a dicing saw (Disco DAD 321). The diced chips were cleaned by a standard RCA procedure as described here. Warning before commencing any experimental work: HF (aq), and hot solutions containing $30 \% \mathrm{H}_{2} \mathrm{O}_{2}$, ammonium hydroxide, and hydrochloric acid must be handled with extreme caution; appropriate PPE, including a face shield, is necessary. The silicon chips were sonicated first in 2-propanol for $10 \mathrm{~min}$, then immersed in a fresh solution of $\mathrm{H}_{2} \mathrm{O} / 30 \% \mathrm{NH}_{4} \mathrm{OH}$ (aq) $/ 30 \% \mathrm{H}_{2} \mathrm{O}_{2}$ (aq) $(6: 1: 1)$ at $80{ }^{\circ} \mathrm{C}$ for $10 \mathrm{~min}$, and next in a fresh solution of $\mathrm{H}_{2} \mathrm{O} / 37 \% \mathrm{HCl}$ (aq) $/ 30 \% \mathrm{H}_{2} \mathrm{O}_{2}$ (aq) $(5: 1: 1)$ at $80{ }^{\circ} \mathrm{C}$ for another $10 \mathrm{~min}$. The chips were rinsed thoroughly with deionized water between each step. The chips were finally rinsed with water and dried with a stream of argon gas. The cleaned silicon chips were immersed in argon-saturated $40 \% \mathrm{NH}_{4} \mathrm{~F}$ for $5 \mathrm{~min}$ and then dipped into deionized water for $10 \mathrm{~s}$. After being dried with an argon stream, each chip was put into an argon-filled vial, sealed, and immediately transferred into a glovebox.

Reactions with Small Molecules. All reactions, except for the etching of silicon surfaces, were performed in the argon-filled glovebox. All the liquid molecules, including 1-octene, 1dodecene, 1-octadecene and 11-fluoro-1-undecene, were passed through a fresh column of dried alumina before use to remove peroxides. Figures 1a-c shows reaction schemes of the hydrideterminated $\operatorname{Si}(111)$ surfaces with three different pure alkenes, a fluoro-terminated alkene and di$n$-octadecyl disulfide. Three methods were used to obtain the monolayer-grafted silicon surfaces, including direct heating using neat molecules, UV irradiation and direct heating with molecules diluted in mesitylene, as shown in Figures 1d-f. After the reaction, the silicon samples were soaked in dry dichloromethane for $5 \mathrm{~min}$ and rinsed three times with a forceful stream of dry dichloromethane from a pipet to remove excess unreacted reagents. The samples were removed from the glovebox (in the sealed glass vial), dried with argon gas, and then analyzed immediately. 
(1) Direct Heating with Neat Alkenes. Twenty drops of liquid reagent were placed on a freshly $\mathrm{NH}_{4} \mathrm{~F}$-etched flat $\mathrm{Si}(111)$ substrate inside a $20-\mathrm{mL}$ glass vial, as shown in Figure 1d. The face of the silicon with the etched side was placed upside down to enforce the spreading of the reagent, thus forming a sandwich of glass-reagent-etched silicon. Then, the glass vials were sealed with multilayers of parafilm entwined around the cap to seal it. The sealed glass vial containing the reagent and the porous silicon substrate was put on a $200 \mathrm{C}$ hot plate for a desired reaction time.

(2) UV Irradiation. As shown previously by our group in 2012, electron acceptors such as chlorobenzene $(\mathrm{PhCl})$ bring about a 200 -fold increase of rate of the pseudo first order rate constant for UV-mediated hydrosilylation alkenes, compared to the rate for neat alkenes. ${ }^{63} \mathrm{Here}, \mathrm{PhCl}$ was dissolved first into the neat alkene of interest to yield a solution with a concentration of $0.25 \mathrm{M} \mathrm{PhCl}$. Approximately $5 \mu \mathrm{L}$ of the alkene $/ \mathrm{PhCl}$ solution was placed on the silicon wafer, which then was covered with a quartz disc, forming a sandwich of quartz coverslip-reagent(s)-silicon wafer (Figure 1e). The quartz disc minimized concentration changes due to evaporation and prevented dewetting of the surface as the monolayer yield increased. Next, the samples were irradiated with a UVP Pen-Ray lamp (254 nm, model 11SC-1) that was held $1 \mathrm{~cm}$ above the samples for various lengths of time. The intensity of the $254 \mathrm{~nm}$ light reaching the quartz surface was measured to be $\sim 2 \mathrm{~mW} / \mathrm{cm}^{2}$.

(3) Direct Heating with Diluted Alkenes. For less common (more expensive) reagents such as 11-fluoro-1-undecene, the alkenes were diluted with mesitylene as per Südholter et al. ${ }^{59}$ As shown in Figure 1f, the alkene of interest was dissolved in mesitylene at a reagent to a solvent dilution ratio of $1: 9$, and about $400 \mu \mathrm{L}$ of the solution was placed on a freshly etched silicon wafer inside a $20-\mathrm{mL}$ glass vial, and the vial was capped and sealed with Parafilm and put on a $200{ }^{\circ} \mathrm{C}$ hot plate for a desired reaction time.

Analytical Techniques. The intensity of the $254 \mathrm{~nm}$ UV light was measured by a Solo 2 energy and power meter with a XLPF12-3S-H2-D0 head (Gentec Electro-Optics Inc.). Sessile drop contact angles and advancing and receding contact angle measurements were taken on an FTA200 video system. X-ray photoelectron spectroscopy (XPS) spectra were taken on a Kratos Axis Ultra X-ray photoelectron spectroscopy system with a power of $14 \mathrm{kV}, 12 \mathrm{~mA}$, and $168 \mathrm{~W}$ using an $\mathrm{Al}$ source with an energy of $1487 \mathrm{eV}$, in the University of Alberta NanoFAB, with 
binding energies calibrated to $\mathrm{Au} 4 \mathrm{f}_{7 / 2}=84.0 \mathrm{eV}$. Photoelectrons were collected using a takeoff angle of $90^{\circ}$ relative to the sample surface.

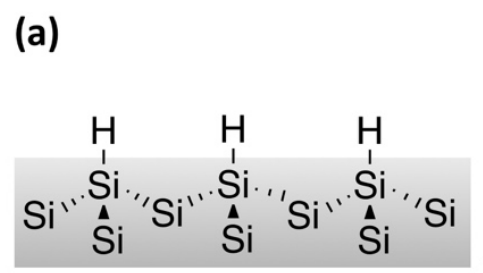

Hydride-terminated $\mathrm{Si}(111)$

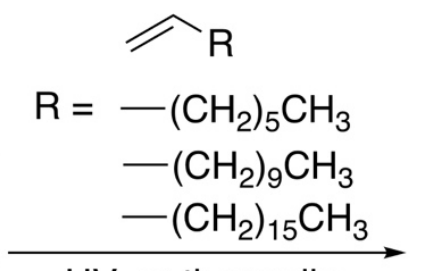

UV- or thermally mediated<smiles>[R]CC[Si]([Si])([Si])[Si][Si][Si]([Si])([Si])[Si][Si][Si]</smiles>

Si(111) surface

(b)

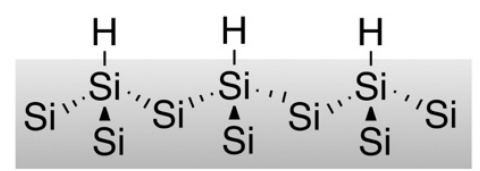

Hydride-terminated $\mathrm{Si}(111)$

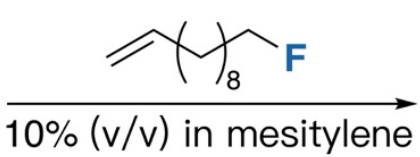

$200{ }^{\circ} \mathrm{C}$

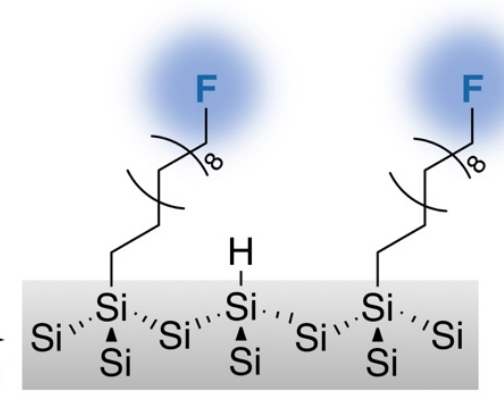

$\mathrm{Si}(111)$ surface

(c)

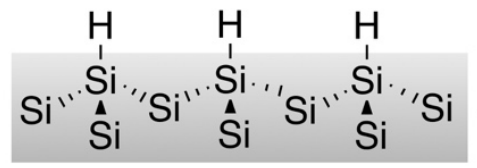

Hydride-terminated $\mathrm{Si}(111)$

(d)

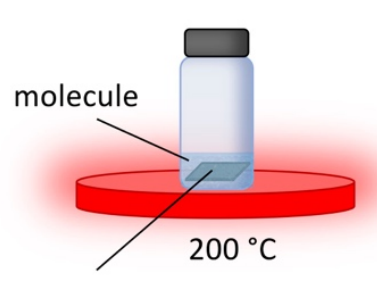

silicon wafer

(e)

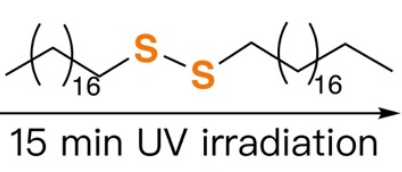

$80{ }^{\circ} \mathrm{C}$

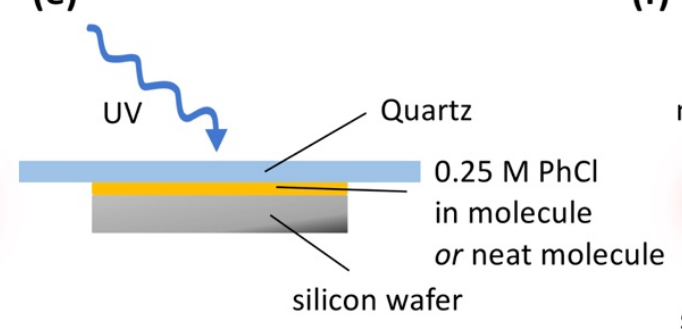

(f)

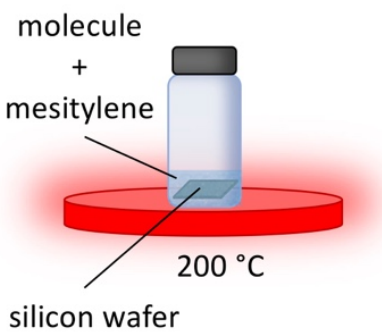

Figure 1. Reaction of $\mathrm{Si}(111)-\mathrm{H}$ with (a) neat alkenes, (b) 11-fluoro-1-undecene diluted in mesitylene, and (c) di- $n$-octadecyl disulfide. Overview of experimental setup for reaction of $\mathrm{Si}(111)-\mathrm{H}$ with an alkene via (d) direct heating using neat molecules, (e) UV irradiation, and (f) direct heating with an alkene diluted in mesitylene. 


\section{- Results}

The quantification of substitution levels of $\equiv \mathrm{Si}(111)-\mathrm{H}$ groups by alkyl groups through the use of calculations based on XPS relies upon a continuum overlayer-substrate model that assumes that each layer has a uniform atomic density, electron attenuation coefficient and thickness. The intensities of photoemitted electrons are attenuated as they pass through a material, and can be calculated according to the Beer-Lambert law. Conventionally, the analysis is typically limited to the carbon and silicon photoelectron signals when one uses XPS to determine substitution levels. However, in addition to the attached alkyl groups on the silicon surface, adventitious carbon also contributes to the $\mathrm{C} 1 \mathrm{~s}$ peak and thus may increase the calculated surface coverage. Here, we take a closer look at the assumptions made with regards to quantifying substitution levels of silicon hydrides on $\mathrm{Si}(111)-\mathrm{H}$ surfaces by alkyl groups, and we develop new models and formulae to adjust these assumptions to enable a quantification that may be more accurate. We will also show how the derived models and equations can be adjusted for $\mathrm{Si}(100)$ surfaces. Three different families of molecules that generate organic monolayers were used in this study, including simple aliphatic alkene molecules with different carbon chain lengths, 1-octene $\left[\mathrm{CH}_{2}=\mathrm{CH}-\left(\mathrm{CH}_{2}\right)_{5}-\mathrm{CH}_{3}\right]$, 1-dodecene $\left[\mathrm{CH}_{2}=\mathrm{CH}-\left(\mathrm{CH}_{2}\right)_{9}-\mathrm{CH}_{3}\right]$ and 1-octadecene $\left[\mathrm{CH}_{2}=\mathrm{CH}-\right.$ $\left.\left(\mathrm{CH}_{2}\right)_{15}-\mathrm{CH}_{3}\right]$, an alkene with an omega-fluorine label, 11-fluoro-1-undecene $\left[\mathrm{CH}_{2}=\mathrm{CH}-\left(\mathrm{CH}_{2}\right)_{9}-\right.$ F], and a non-alkene disulfide that ends up bound to the surface through the sulfur atom, di- $n$ octadecyl disulfide $\left[\mathrm{CH}_{3}-\left(\mathrm{CH}_{2}\right)_{17}-\mathrm{SS}-\left(\mathrm{CH}_{2}\right)_{17}-\mathrm{CH}_{3}\right]$. Conventional reaction conditions, including direct thermal heating and UV irradiation with neat molecules or molecules dissolved in mesitylene, were employed to functionalize the flat $\mathrm{Si}(111)-\mathrm{H}$ surface with alkenes. For di- $n$ octadecyl disulfide, the radical-based functionalization method and XPS data used for quantification were previously published by our group. ${ }^{43}$

Contact Angle Analysis. To ensure that the monolayers produced via alkene hydrosilylation or reaction with the dialkyl disulfide reached their maximum substitution level for the given reaction conditions, the functionalized silicon surfaces were first analyzed by goniometry (water contact angle measurements, Table 1 and Figure 2). The static contact angle for the starting hydride-terminated $\mathrm{Si}(111)$ surface is $\sim 83^{\circ}$. After reaction with 1-octene, 1-dodecene and 1octadecene, the maximum contact angles measured were $\sim 103^{\circ}, \sim 102^{\circ}$ and $\sim 106^{\circ}$, respectively; these values are similar to those of other silicon surfaces functionalized with long alkyl chains 
from the literature. ${ }^{59-62}$ For the $\equiv \mathrm{Si}-\left(\mathrm{CH}_{2}\right)_{11}-\mathrm{F}$ surface obtained via refluxing in a $10 \%$ solution in mesitylene, the water contact angle is $\sim 93^{\circ}$, similar to that previously observed for $\equiv \mathrm{Si}-$ $\left(\mathrm{CH}_{2}\right)_{10}-\mathrm{F}$ on $\mathrm{Si}(111)\left(\sim 94^{\circ}\right) .{ }^{41}$ Water contact angles are useful to determine reaction kinetics to ensure completion, ${ }^{63}$ as shown in Figure 2 for UV-mediated irradiation of 1-octadecene, which appear to follow classic Langmuir kinetics, ${ }^{64}$ with a maximum contact angle of $105^{\circ}$ reached after $45 \mathrm{~s}$.

Table 2. Static Contact Angles for the Functionalized Si(111)-H Surfaces Under Different Reaction Conditions

\begin{tabular}{|c|c|c|c|}
\hline Reagent & Reaction Conditions & $\theta_{\mathrm{e}}^{\mathrm{w} a}\left({ }^{\circ}\right)$ & $\theta_{\mathrm{a}}{ }^{\mathrm{w} a}\left({ }^{\circ}\right) / \theta_{\mathrm{r}}{ }^{\mathrm{w} a}\left({ }^{\circ}\right)$ \\
\hline $\mathrm{Si}(111)-\mathrm{H}$ surface & N/A & $83.3 \pm 0.9$ & $95 \pm 0.8 / 75 \pm 0.8$ \\
\hline 1-Octene & $10 \%$ in mesitylene, $200{ }^{\circ} \mathrm{C}, 2 \mathrm{~h}$ & $103.3 \pm 1.9$ & $105 \pm 0.4 / 100 \pm 0.2$ \\
\hline 1-Dodecene & $10 \%$ in mesitylene, $200{ }^{\circ} \mathrm{C}, 2 \mathrm{~h}$ & $102.3 \pm 1.3$ & $109 \pm 1.0 / 101 \pm 0.9$ \\
\hline \multirow[t]{3}{*}{ 1-Octadecene } & Excess neat molecule, $200^{\circ} \mathrm{C}, 2 \mathrm{~h}$ & $105.4 \pm 1.3$ & $110 \pm 0.8 / 100 \pm 0.4$ \\
\hline & $0.25 \mathrm{M} \mathrm{PhCl}$ in alkene, $\mathrm{UV}, 1 \mathrm{~min}$ & $105.4 \pm 0.8$ & $109 \pm 1.0 / 100 \pm 0.9$ \\
\hline & $\begin{array}{l}10 \% \text { alkene in mesitylene, } 200^{\circ} \mathrm{C}, 2 \\
\mathrm{~h}\end{array}$ & $106.0 \pm 0.9$ & $112 \pm 0.5 / 98 \pm 0.7$ \\
\hline \multirow[t]{2}{*}{ 11-Fluoro-1-undecene } & $0.25 \mathrm{M} \mathrm{PhCl}$ in alkene, $\mathrm{UV}, 1 \mathrm{~min}$ & $88.4 \pm 1.2$ & $94 \pm 0.9 / 87 \pm 0.4$ \\
\hline & $\begin{array}{l}10 \% \text { alkene in mesitylene, } 200^{\circ} \mathrm{C}, 2 \\
\mathrm{~h}\end{array}$ & $93.0 \pm 1.4$ & $95 \pm 0.6 / 90 \pm 0.2$ \\
\hline Di- $n$-octadecyl disulfide ${ }^{b}$ & $\mathrm{UV}, 15 \mathrm{~min}, 80^{\circ} \mathrm{C}$ & $105.1 \pm 0.4$ & $108 \pm 1.0 / 88 \pm 0.8$ \\
\hline
\end{tabular}

${ }^{a} \mathrm{e}$, a, and $\mathrm{r}$ refer to equilibrium, advancing, and receding, respectively, and $\mathrm{w}$ and HD represent water and hexadecane, respectively. Each value is the average of five separate measurements, and the error listed is the standard deviation. ${ }^{b}$ The data for di- $n$-octadecyl disulfide-functionalized silicon surface has been previously published (reference 43). 


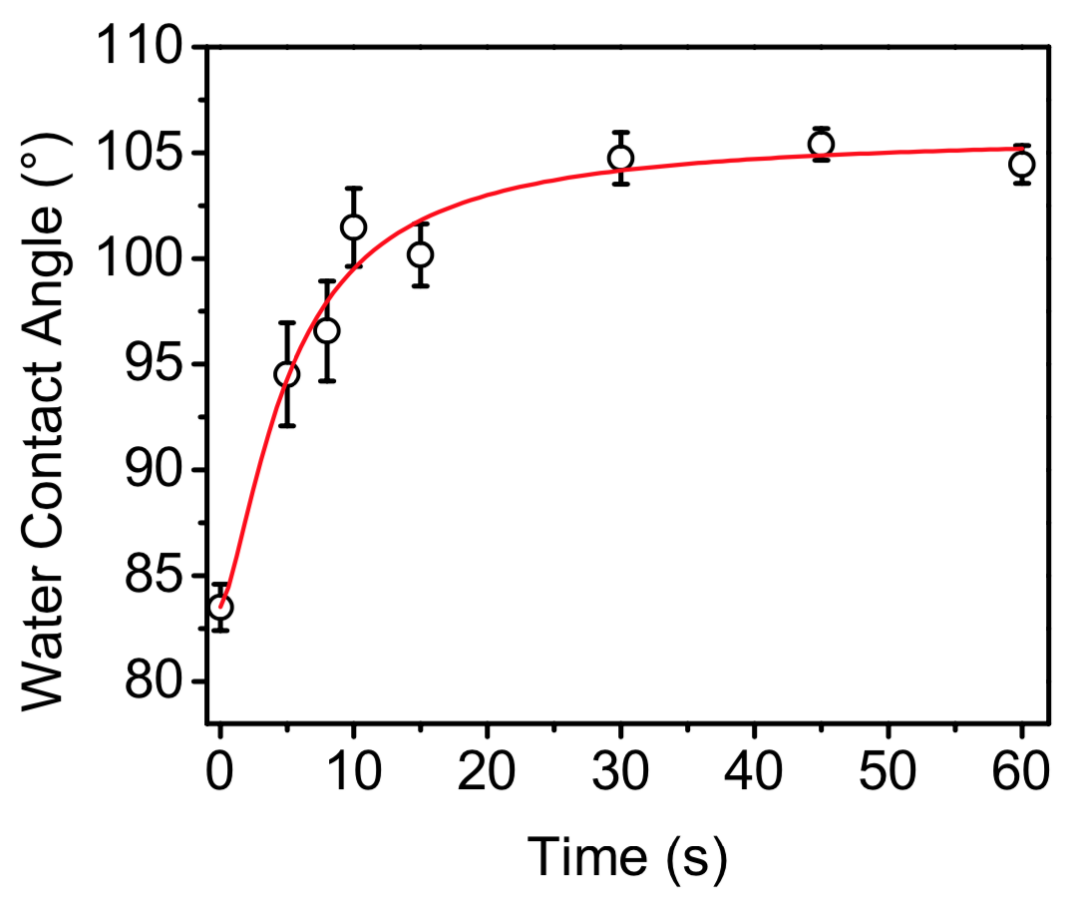

Figure 2. Kinetic profile of hydrosilylation of 1-octadecene on $\mathrm{Si}(111)-\mathrm{H}$ with $0.25 \mathrm{M} \mathrm{PhCl}$ and UV irradiation, as observed via goniometry (static water contact angle measurements). Each black dot represents a unique sample that had been reacted for the indicated reaction time. The error bars represent the standard deviation of five measurements on the same sample. The red curve is a fit to the first order Langmuir rate equation. ${ }^{64}$

X-ray Photoelectron Spectroscopy. XPS was carried out on Si(111)-H samples reacted with 1octene, 1-dodecene, 1-octadecene, and 11-fluoro-1-undecene, as shown in Figure 3. Highresolution Si $2 p$ and C 1s XPS data were analyzed using CasaXPS version 2.3.17. Spectra were analyzed using a Shirley background. Si 2 p spectra were deconvoluted to two sets of peaks, where the blue set represents the Si $2 p$ photoelectron signals from the bulk silicon and the red one set represents the Si $2 p$ photoelectron signals from the surface. Bulk Si $2 p$ data were fitted using an asymmetric Gaussian-Lorentz lineshape, implemented in CasaXPS as LA $(a, b, m)$, where the ratio of $a$ and $b$ define the asymmetry of the Lorentzian profile and $m$ is an integer that defines the width of the Gaussian that is convolved with the Lorentzian. $\operatorname{LA}(2,3.2,300)$ was used in this work. C 1s spectra and contributions from the surface Si $2 p$ were fitted using the GL(30) line shape, which consisted of 70\% Gaussian and 30\% Lorentzian character. Spin-orbit components were constrained to have the same full width at half maximum (FWHM). The Si $2 p$ spin-orbit splitting was fixed to $0.63 \mathrm{eV}$, and the $\mathrm{Si} 2 \mathrm{p}_{1 / 2}$ peak was constrained to half the intensity of the Si $2 p_{3 / 2}$ peak. A gold-on-silicon sample was used as the reference for XPS 
calibration (and not the C 1s), ${ }^{65}$ as per Lewis and co-workers. ${ }^{66}$ The Si $2 p$ spectra of all four silicon samples reveal no significant oxidation, which would appear as higher energy features above $102 \mathrm{eV}$ due to the oxygen insertion into surface $\mathrm{Si}-\mathrm{Si}$ and $\mathrm{Si}-\mathrm{H}_{x}$ bonds. ${ }^{67,68}$ For the models and subsequent equations derived in this work it is assumed that oxidation of the silicon surface is negligible. As such, silicon surfaces that possess these higher binding energy features in the $\mathrm{Si}$ $2 p$ spectra are not included in the framework of this paper. All four $\mathrm{C} 1 \mathrm{~s}$ spectra show the expected features representative of alkyl chains on silicon surfaces, with the main peak at 284.8 $\mathrm{eV}$ corresponding to the carbon in the alkyl chains. The feature at $287.8 \mathrm{eV}$ is assigned to the carbon bound to fluorine for the $\equiv \mathrm{Si}-\left(\mathrm{CH}_{2}\right)_{11}-\mathrm{F}$ surface. XPS data for the four functionalized surfaces are shown in Table 3. 

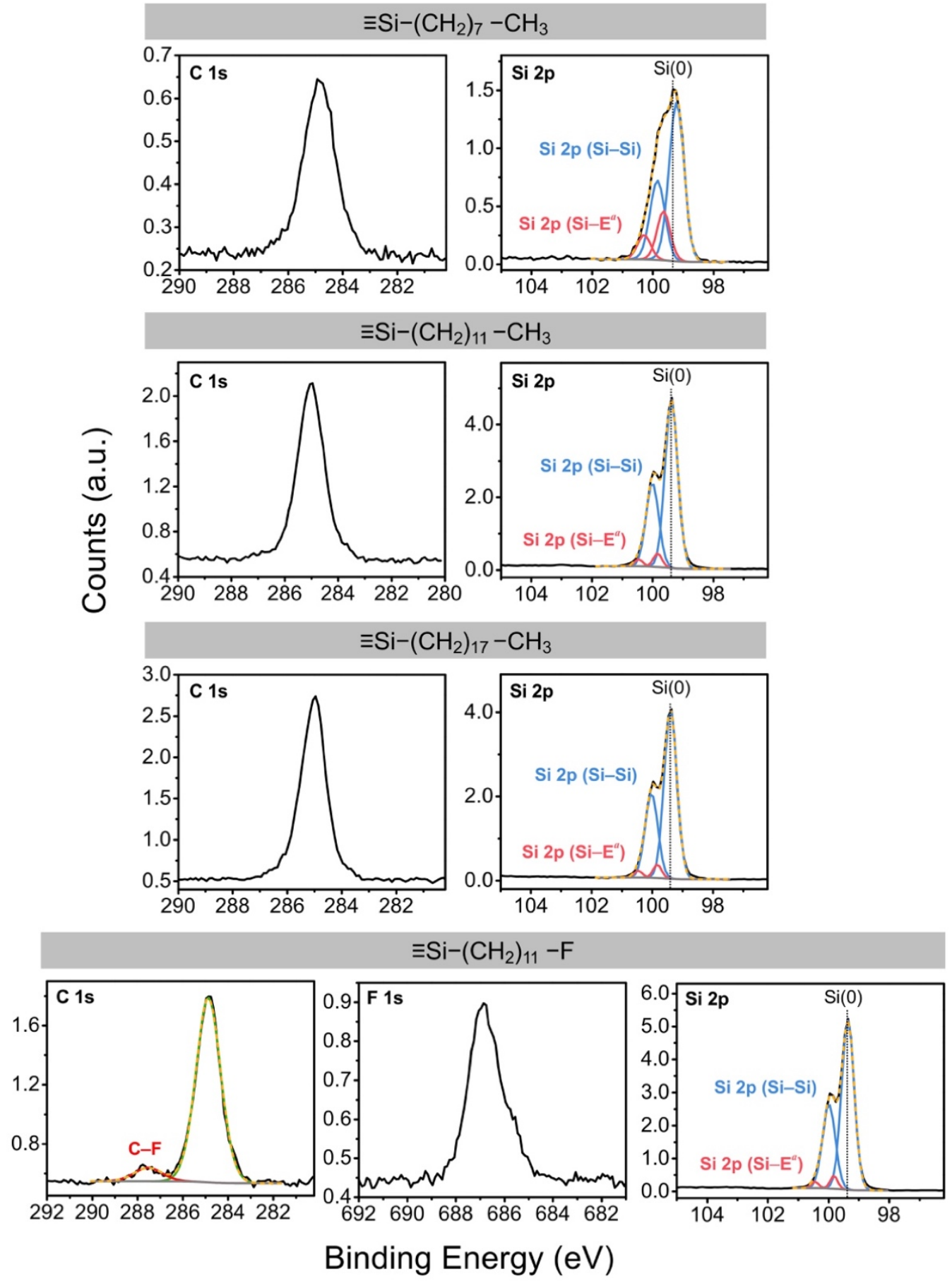

Figure 3. XPS spectra of $\mathrm{Si}(111)-\mathrm{H}$ surfaces after thermal hydrosilylation with 1-octene, 1dodecene, 1-octadecene, and 11-fluoro-1-undecene ( $10 \%$ in mesitylene solutions, v/v) at $200{ }^{\circ} \mathrm{C}$ for $2 \mathrm{~h}$. Yellow dashed curves are envelope fittings. The data from this figure are tabulated in Table 3. $\mathrm{E}^{a}=\mathrm{C}$ and $\mathrm{H}$. 
Table 3. Summary of XPS Data Corresponding to Figure 3

\begin{tabular}{lccccc}
\hline & $\begin{array}{c}\mathrm{Si} 2 \mathrm{p}_{3 / 2} \\
(\mathrm{bulk}, \mathrm{eV})\end{array}$ & $\begin{array}{c}\mathrm{Si}_{2} \mathrm{p}_{3 / 2} \\
(\text { surface, eV })\end{array}$ & $\begin{array}{c}\mathrm{C} 1 \mathrm{~s} \\
(\mathrm{eV})\end{array}$ & $\begin{array}{c}\mathrm{C} \text { 1s }(\mathrm{C}-\mathrm{F}) \\
(\mathrm{eV})\end{array}$ & $\begin{array}{c}\mathrm{F} \text { 1s } \\
(\mathrm{eV})\end{array}$ \\
\hline 1-Octene & 99.20 & 99.64 & 284.8 & & \\
1-Dodecene & 99.37 & 99.83 & 285.0 & & \\
1-Octadecene & 99.39 & 99.83 & 285.0 & & \\
11-Fluoro-1-undecene & 99.36 & 99.83 & 284.9 & 287.5 & 686.8 \\
\hline
\end{tabular}

Previous work by the Zuilhof group using the carbon:silicon ratio via XPS initially converted this ratio into a monolayer thickness, $d_{\mathrm{ML}}$, using the following relationship, ${ }^{36}$

$$
d_{\mathrm{ML}}=\lambda_{\mathrm{ML}} \sin (\theta) \ln (1+\mathrm{C} / \mathrm{Si})
$$

where $\lambda_{\mathrm{ML}}$ is the attenuation length of Si $2 p$ photoelectrons in the organic monolayers $\left(\lambda_{\mathrm{ML}}=39.5\right.$ $\AA)$, and $\theta$ is the takeoff angle between the surface and the detector $\left(\theta=90^{\circ}\right)$. The surface coverage was calculated by comparing the formed monolayers with previous work on alkanethiol monolayer on gold. ${ }^{34,36,37,41}$ The surface coverage, $\varphi$, of the monolayers was given by

$$
\phi=\frac{d_{\mathrm{ML}} \times D_{\mathrm{Au}}}{d_{\mathrm{TH}} \times D_{\mathrm{Si}}}
$$

where $D_{\mathrm{Au}}$ is the surface density of alkanethiols in a perfect SAM on gold with a tilt angle of $30^{\circ}$ $\left(D_{\mathrm{Au}}=4.65 \times 10^{14} \mathrm{~cm}^{-2}\right), D_{\mathrm{Si}}$ is the surface atom density on $\operatorname{Si}(111)\left(D_{\mathrm{Si}}=7.8 \times 10^{14} \mathrm{~cm}^{-2}\right)$, and $d_{\mathrm{TH}}$ is the theoretical thickness of an organic monolayer on Si with an assumed tilt angle of 30$35^{\circ} .36,37$ This model and resulting calculations are simple and straightforward, but they do not consider adventitious or physisorbed hydrocarbons. 
In 2005, Yamazaki and co-workers quantified the surface coverages, $\varphi$, of fluorine- and nitrogen-containing functionalities on $\mathrm{Si}(111)$ surfaces using the relative intensities of $\mathrm{F} 1 \mathrm{~s}$ and $\mathrm{N}$ 1s peaks with respect to $\mathrm{Si} 2 \mathrm{p}$ peaks, while taking adventitious carbon contamination into consideration. ${ }^{35} \mathrm{Eq} 3$ is shown as an example in their work for the estimation of the coverage of a $\mathrm{CF}_{3}$-terminated surface,

$$
\phi=\frac{I_{\mathrm{F}}}{I_{\mathrm{Si}}} \frac{\sigma_{\mathrm{Si}}}{3 \sigma_{\mathrm{F}}} \frac{\exp \left(-d_{\mathrm{ml}} / \lambda_{\mathrm{ml}} \cos \theta\right)}{\left[1-\exp \left(-d_{111}^{\prime} / \lambda_{\mathrm{Si}} \cos \theta\right)\right]}
$$

where $I_{\mathrm{F} 1 \mathrm{~s}}$ and $I_{\mathrm{Si} 2 \mathrm{p}}$ are the signal intensities of $\mathrm{F} 1 \mathrm{~s}$ and $\mathrm{Si} 2 \mathrm{p}$ photoelectrons, respectively. $\sigma_{\mathrm{F}}$ and $\sigma_{\mathrm{Si}}$ are the relative sensitivity factors of the $\mathrm{F} 1 \mathrm{~s}$ and $\mathrm{Si} 2 \mathrm{p}$ photoelectrons. The coefficient of 3 before the $\mathrm{RSF}_{\mathrm{F}}$ term in the Eq 3 is due to the termination of a single chain with three fluorine atoms. $d_{\mathrm{ml}}$ is the thickness of the monolayer on the Si surface, and $d^{\prime}{ }_{111}$ is the average layer spacing, $1.568 \AA$. $\theta$ represents the take-off angle in the XPS measurement, and $\lambda_{\mathrm{Si}}$ and $\lambda_{\mathrm{ml}}$ in their work represent the inelastic mean free path of Si photoelectrons and organic monolayers, respectively. The surface coverage ranges of $54-56 \%, 55-60 \%, 42-67 \%$, and $46-50 \%$ were obtained for $\mathrm{Si}-\mathrm{CH}=\mathrm{CH}-\mathrm{CO}_{2} \mathrm{CH}_{2} \mathrm{CF}_{3}, \mathrm{Si}-\mathrm{CH}=\mathrm{CH}-\mathrm{CO}_{2} \mathrm{CH}_{2}-\mathrm{C}_{6} \mathrm{H}_{4}-p-\mathrm{CF}_{3}, \mathrm{Si}-\mathrm{CH}=\mathrm{CH}-\mathrm{C}_{6} \mathrm{H}_{4}-p$ $\mathrm{CF}_{3}$, and $\mathrm{Si}-\mathrm{CH}=\mathrm{CH}-\mathrm{C}_{6} \mathrm{H}_{4}-p-\mathrm{F}$ surfaces, respectively, at different take-off angles $\left(0^{\circ}, 30^{\circ}\right.$, and $\left.60^{\circ}\right)$. While using a heteroatom as an atomic label yields the most accurate estimation of coverage, one still needs to consider the effect of the changing attenuation length with the change of substitution level (vide infra), and the fact that any adventitious carbon layer would also result in attenuation of signals from the layer underneath.

\section{Reconsideration of the models and calculations used for XPS-based determination of} surface coverage on $\mathrm{Si}(\mathbf{1 1 1})$. Here, we reconsider the model, starting from scratch with a structural profile of the resulting organic monolayers. Schematics of the cross-sectional profiles of the monolayers comprising $\equiv \mathrm{Si}-\left(\mathrm{CH}_{2}\right)_{n}-\mathrm{CH}_{3}\left(\mathrm{n}=7,11\right.$, and 17), $\equiv \mathrm{Si}-\mathrm{S}-\left(\mathrm{CH}_{2}\right)_{17}-\mathrm{CH}_{3} \equiv \mathrm{Si}-$ $\left(\mathrm{CH}_{2}\right)_{11}-\mathrm{F}$, and $\equiv \mathrm{Si}-\mathrm{S}-\left(\mathrm{CH}_{2}\right)_{17}-\mathrm{CH}_{3}$ are shown in Figure 4. The surfaces have been divided into at least three sublayers, starting with the silicon substrate at the bottom, the organic monolayer in the middle, and the possible adventitious carbon layer at the top. For the $\equiv \mathrm{Si}-\left(\mathrm{CH}_{2}\right)_{11}-\mathrm{F}$ surface, the organic layer is further divided into the very thin outermost top monofluorine "layer", and the underlying alkyl layer. Similarly, the organic layer of the $\equiv \mathrm{Si}-\mathrm{S}-\left(\mathrm{CH}_{2}\right)_{17}-\mathrm{CH}_{3}$ surface is 
composed of the overlying alkyl layer and the thin "layer" comprising the sulfur atoms attached to the silicon surface. Quantitative XPS data of the 5 silicon surfaces are shown in Table 4.
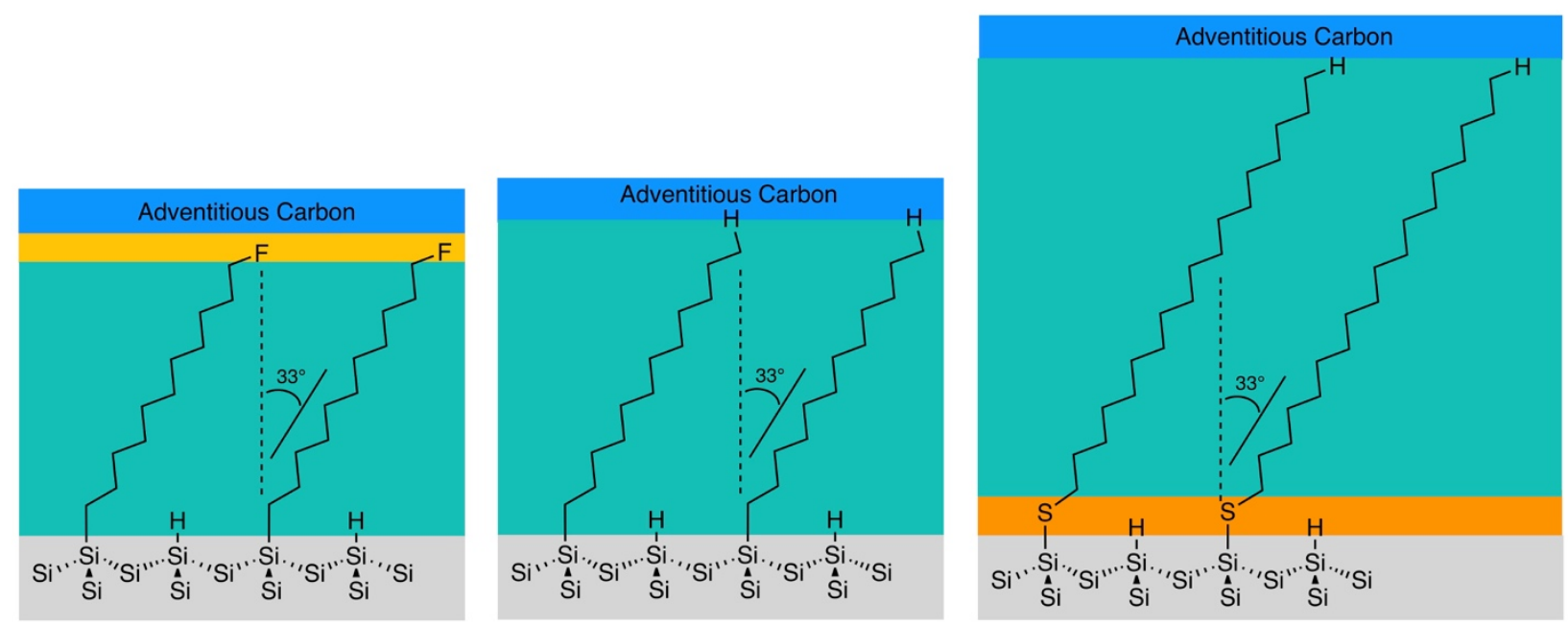

Figure 4. Schematic models of the the $\equiv \mathrm{Si}(111)-\left(\mathrm{CH}_{2}\right)_{11} \mathrm{~F}$, $\equiv \mathrm{Si}-\left(\mathrm{CH}_{2}\right)_{\mathrm{n}} \mathrm{CH}_{3}$, and $\equiv \mathrm{Si}-\mathrm{S}-\left(\mathrm{CH}_{2}\right)_{17} \mathrm{CH}_{3}$ surfaces, from left to right, respectively.

Table 4. Quantitative XPS Data for Indicated Functionalized Si(111)-H Surfaces

\begin{tabular}{lccc}
\hline Surface & $\mathrm{C} / \mathrm{Si}$ ratio & F/Si ratio & S/Si ratio \\
\hline$\equiv \mathrm{Si}-\left(\mathrm{CH}_{2}\right)_{11}-\mathrm{F}$ & 0.441 & 0.187 & -- \\
$\equiv \mathrm{Si}-\left(\mathrm{CH}_{2}\right)_{7}-\mathrm{CH}_{3}$ & 0.362 & -- & -- \\
$\equiv \mathrm{Si}-\left(\mathrm{CH}_{2}\right)_{11}-\mathrm{CH}_{3}$ & 0.523 & -- & -- \\
$\equiv \mathrm{Si}-\left(\mathrm{CH}_{2}\right)_{17}-\mathrm{CH}_{3}$ & 0.790 & -- & -- \\
$\equiv \mathrm{Si}-\mathrm{S}-\left(\mathrm{CH}_{2}\right)_{17}-\mathrm{CH}_{3}$ & 0.749 & -- & 0.052 \\
\hline
\end{tabular}

Quantification of substitution level using XPS requires careful consideration of the attenuation length in the organic films. SAMs comprising $n$-alkanethiols on gold are the most widely studied system of densely packed, oriented molecules on surfaces. ${ }^{69-73}$ The thickness of the films can be easily controlled by changing the length of the aliphatic chain. Whitesides and co-workers benchmarked the attenuation length of electrons in these SAMS at selected kinetic energies using angle-resolved XPS measurements, ${ }^{74}$ and derived the following empirical formula that connects 
the attenuation lengths of electrons $\left(\lambda_{\mathrm{ML}}{ }^{\mathrm{Au}}\right)$, emitted through the organic monolayers on the gold surface as a function of kinetic energy $\left(E_{k}\right)^{75}$

$$
\lambda_{\mathrm{ML}}^{\mathrm{Au}}=9+0.022 E_{\mathrm{k}}
$$

Using the formula above, the attenuation length of a Si $2 \mathrm{p}$ photoelectron can be estimated, with the following variations. ${ }^{76}$ An ideal $2 \times 1$ structure on $\mathrm{Si}(111)$ would be $16 \%$ less dense than a $(\sqrt{ } 3 \times \sqrt{ } 3) R 30^{\circ}$ n-alkanethiol SAM on $\mathrm{Au}(111),{ }^{34}$ and thus the attenuation length of a Si $2 p$ photoelectron travelling through a hydrocarbon monolayer on silicon, $\lambda_{\mathrm{SiC}}{ }^{\mathrm{Si}}$, would be larger than that of gold, $\lambda_{\mathrm{SiC}^{\mathrm{Au}}}$. Although the value of $\lambda_{\mathrm{SiC}} \mathrm{Si}^{\mathrm{S}}$ is not available experimentally, it can be estimated by scaling the values for gold surfaces using the relationship between attenuation length and atomic density on silicon and gold, as will be described in the next section.

Connection between Attenuation Length and Atomic Density on $\mathrm{Si}(111)$ and $\mathrm{Au}(111)$. To estimate the value of $\lambda_{\mathrm{SiC}}{ }^{\mathrm{Si}}$, the scaling values for gold surfaces were used as a starting point. According to Tanuma and co-workers, ${ }^{77}$ for an electron emitted from element $x$, going through a material $y$ with a structure $\alpha$, the product of the attenuation coefficient, $\lambda_{x y}^{\alpha}$, multiplied by atomic density, $\rho_{y}^{\alpha}$, will be a constant, specifically if the electron is travelling through a different structure, $\beta$, of element $y$, that has a different density, $\rho_{y}^{\beta}$

$$
\lambda_{x y}^{\alpha} \rho_{y}^{\alpha}=\lambda_{x y}^{\beta} \rho_{y}^{\beta}
$$

When Eq 3 was applied to the case of the attenuation length of an element ( $\mathrm{Si}$ or $\mathrm{C}$ ) on silicon and gold substrates, the attenuation length of an electron emitted from Si, travelling through an organic carbon layer on a silicon substrate, $\lambda_{\mathrm{SiC}}^{\mathrm{Si}}$, is given by

$$
\begin{gathered}
\lambda_{\mathrm{SiC}}^{\mathrm{Si}} \rho_{\mathrm{C}}^{\mathrm{Si}}=\lambda_{\mathrm{SiC}}^{\mathrm{Au}} \rho_{\mathrm{C}}^{\mathrm{Au}} \\
\lambda_{\mathrm{SiC}}^{\mathrm{Si}}=\frac{\rho_{\mathrm{C}}^{\mathrm{Au}}}{\rho_{\mathrm{C}}^{\mathrm{Si}}} \lambda_{\mathrm{SiC}}^{\mathrm{Au}}
\end{gathered}
$$


The attenuation length of Si going through the adventitious carbon layer on a silicon substrate, $\lambda_{\mathrm{SiA}}$, is given by

$$
\lambda_{\mathrm{SiA}}=\frac{\rho_{\mathrm{C}}^{\mathrm{Au}}}{\rho_{\mathrm{A}}} \lambda_{\mathrm{SiC}}^{\mathrm{Au}}
$$

The attenuation length of $\mathrm{C}$ going through the organic carbon layer on a silicon substrate, $\lambda_{\mathrm{CC}}^{\mathrm{Si}}$, is given by

$$
\lambda_{\mathrm{CC}}^{\mathrm{Si}}=\frac{\rho_{\mathrm{C}}^{\mathrm{Au}}}{\rho_{\mathrm{C}}^{\mathrm{Si}}} \lambda_{\mathrm{CC}}^{\mathrm{Au}}
$$

The attenuation length of $\mathrm{C}$ going through the adventitious carbon layer on a silicon substrate, $\lambda_{\mathrm{CA}}$, is given by

$$
\lambda_{\mathrm{CA}}=\frac{\rho_{\mathrm{C}}^{\mathrm{Au}}}{\rho_{\mathrm{A}}} \lambda_{\mathrm{CC}}^{\mathrm{Au}}
$$

The obtained equations, 6, 7, 8 and 9, show that the attenuation length for an electron derived from $\mathrm{Si}$ or $\mathrm{C}$ passing through a specific carbon layer (the carbon within the alkyl chains in the monolayer, or adventitious carbon) on a silicon substrate can be estimated as the product of the attenuation length on a gold substrate multiplied by the ratio of the atomic density of carbon on gold relative to carbon's atomic density on silicon. These equations will be used in the following section regarding quantification of the substitution levels. 
Calculations of Constants. This section describes the detailed calculations of the constants that will be applied in the quantification of substitution levels for all the functionalized silicon surfaces, including the atomic density (carbon atoms) of the hydrocarbon layer on a gold substrate, $\rho_{\mathrm{C}}^{\mathrm{Au}}$, the attenuation length of the Si $2 \mathrm{p}$ electron travelling through an alkyl monolayer on gold, $\lambda_{\mathrm{SiC}^{\mathrm{Au}}}$, and the attenuation length of $\mathrm{C} 1 \mathrm{~s}$ electron in an alkyl monolayer on gold, $\lambda_{\mathrm{CC}}{ }^{\mathrm{Au}}$. The atomic density of carbon-on-gold, $\rho_{\mathrm{C}}^{\mathrm{Au}}$, can be calculated by assuming that the organic layer would have the same surface density as the surface of alkanethiols in a perfect SAM on $\mathrm{Au}, n_{\mathrm{Au}}$ $\left(4.6 \times 10^{14}\right.$ atoms $\left./ \mathrm{cm}^{2}\right)$, and dividing that by the average thickness per carbon atomic plane or the theoretical thickness $(1.32 \mathrm{~nm})$ of each chain normalized by the number of carbons per chain, $N_{\text {chain }}(12)$.

$$
\rho_{\mathrm{C}}^{\mathrm{Au}}=\frac{n_{\mathrm{Au}}}{d_{\mathrm{C}}^{\mathrm{Au}} / N_{\text {chain }}}=\frac{4.6 \times 10^{14}}{1.32 \times 10^{-7} / 12}=4.1 \times 10^{22} \text { atoms } / \mathrm{cm}^{3}
$$

The atomic density of carbon on the silicon substrate, $\rho_{\mathrm{C}}^{\mathrm{Si}}$, is given by

$$
\rho_{\mathrm{C}}^{\mathrm{Si}}=\frac{\phi n_{\mathrm{Si}}}{d_{\mathrm{C}}^{\mathrm{Si}} / N_{\text {chain }}}=\frac{\phi n_{\mathrm{Si}} N_{\text {chain }}}{d_{\mathrm{C}}^{\mathrm{Si}}}
$$

where $n_{\mathrm{Si}}$ is the atomic surface density of silicon.

As mentioned before, the attenuation length of photoelectrons in alkyl monolayers on gold, $\lambda_{\mathrm{ML}}^{A u}$, can be calculated using the empirical formula described by the Whitesides et al

$$
\lambda_{\mathrm{ML}}^{\mathrm{Au}}=9+0.022 E_{\mathrm{k}}
$$

where $E_{\mathrm{k}}$ is the kinetic energy of a specific element (measured in $\mathrm{eV}$ ), and the result is in $\AA$. Therefore, the attenuation length of Si $2 p$ electrons in an alkyl monolayer on gold is given by

$$
\lambda_{\mathrm{SiC}}^{\mathrm{Au}}=9+0.022 E_{\mathrm{k}}=9+0.022(1487-99.4 \mathrm{eV})=39.5 \AA
$$

The attenuation length of $\mathrm{C} 1 \mathrm{~s}$ electrons in an alkyl monolayer on gold is given by 


$$
\lambda_{\mathrm{CC}}^{\mathrm{Au}}=9+0.022 E_{\mathrm{k}}=9+0.022(1487-284.8 \mathrm{eV})=35.4 \AA
$$

The attenuation length of $\mathrm{F} 1 \mathrm{~s}$ electrons in an alkyl monolayer on gold is given by

$$
\lambda_{\mathrm{FC}}^{\mathrm{Au}}=9+0.022 E_{\mathrm{k}}=9+0.022(1487-686.8 \mathrm{eV})=26.6 \AA
$$

At this point, we have calculated the attenuation length for $\mathrm{C}$ (in the form of the alkyl chains) on silicon, $\lambda_{\mathrm{CC}}^{\mathrm{Si}} . \lambda_{\mathrm{CC}}^{\mathrm{Si}}$ equals the attenuation length of carbon on gold, $\lambda_{\mathrm{CC}}^{\mathrm{Au}}(\mathrm{a}$ constant), multiplied by the ratio of the atomic density of carbon on gold to the atomic density of carbon on silicon, $\frac{\rho_{\mathrm{C}}^{\mathrm{Au}}}{\rho_{\mathrm{C}}^{\mathrm{Si}}}$, i.e. $\lambda_{\mathrm{CC}}^{\mathrm{Si}}=\frac{\rho_{\mathrm{C}}^{\mathrm{Au}}}{\rho_{\mathrm{C}}^{\mathrm{Si}}} \lambda_{\mathrm{CC}}^{\mathrm{Au}}$.

All constants are listed in Table 5. These values are used in the model of alkyl-based monolayers on silicon.

Table 5. List of Constants Used for the Calculation of Substitution Levels on Si(111)-H from XPS Data

\begin{tabular}{c|c}
\hline Constant & Value \\
\hline $\begin{array}{c}\text { Sensitivity factor } \\
\sigma_{\mathrm{Si}}, \sigma_{\mathrm{F}}, \sigma_{\mathrm{C}}\end{array}$ & $0.328,1.000,0.278$ \\
\hline $\begin{array}{c}\text { Atomic surface density } \\
n_{\mathrm{Si}},{ }^{a} n_{\mathrm{Au}}\end{array}$ & $7.8 \times 10^{14}$ atoms $/ \mathrm{cm}^{2}, 4.6 \times 10^{14}$ atoms $/ \mathrm{cm}^{2}$ \\
\hline $\begin{array}{c}\text { Attenuation coefficients } \\
\lambda_{\mathrm{SiSi}},{ }^{b} \lambda_{\mathrm{SiC}}^{\mathrm{Au}},{ }^{2} \lambda_{\mathrm{CC}}^{\mathrm{Au}},{ }^{b} \lambda_{\mathrm{FC}}^{\mathrm{Au}}\end{array}$ & $1.8 \mathrm{~nm}, 3.95 \mathrm{~nm}, 3.54 \mathrm{~nm}, 2.66 \mathrm{~nm}$ \\
\hline $\begin{array}{c}\text { Atomic density } \\
\rho_{\mathrm{Si}},{ }^{c} \rho_{\mathrm{C}}^{\mathrm{Au}}\end{array}$ & $5 \times 10^{22}$ atoms $/ \mathrm{cm}^{3}, 4.1 \times 10^{22}$ atoms $/ \mathrm{cm}^{3}$ \\
\hline
\end{tabular}

${ }^{a}(\sqrt{ } 3 \times \sqrt{ } 3) R 30$ surface of $(111) .{ }^{b}$ Calculated based on an empirical formula from Whitesides and co-workers. ${ }^{75}$ ${ }^{c}$ Calculated based on the gold surface.

\section{Monolayer with an $\omega$-fluorine tag: the $\equiv \mathrm{Si}-\left(\mathrm{CH}_{2}\right)_{11}-\mathrm{F}$ surface.}

Yamazaki and co-workers relied on a heteroatom tag as the XPS label for the quantification of the surface coverage of activated alkynes on $\mathrm{Si}(111)-\mathrm{H}$, as shown in Eq 3, which is an effective 
way to avoid the error arising from the adventitious carbon on the surface. ${ }^{35}$ However, the change of the surface coverage leads to a change of the attenuation length of Si photoelectrons in the organic carbon layer, which needs to be considered when one quantifies the coverage. Moreover, adventitious carbon can be regarded as an extra thin carbon layer formed on the selfassembled monolayers, with a certain atomic density and thickness. This adventitious carbon layer will also attenuate the signals of photoelectrons emitted from the layers underneath. Here, in our work, because of the presence of multiple layers as shown schematically in Figure 4, a continuum substrate-overlayer model was used to determine the substitution level, $\varphi$, of the alkyl overlayer on silicon, where it was assumed that each layer had a uniform atomic density, $\rho_{i}$, electron attenuation coefficient, $\lambda_{i}$, and thickness, $d_{i}$. It is worth noting that the attenuation length of Si penetrating the organic carbon layer, $\lambda_{\mathrm{SiC}}^{\mathrm{Si}}$, is no longer a constant but a function of the atomic density of carbon on the silicon substrate, which depends on the substitution level and also has taken the tilt angle into consideration (vide infra). ${ }^{76}$ Conventionally, the substitution level is obtained by dividing the effective thickness, $d$, by the theoretical monolayer thickness, $d_{\mathrm{TH}}$. Here in our revised formulae, the thickness of the alkyl layer no longer needs to be calculated because the product of thickness and density is found in all the equations, which is equal to $\varphi \times n_{\mathrm{Si}} \times N_{\text {chain, }}$, as per Eq.10; the substitution levels will be calculated directly from the revised formulae (vide infra).

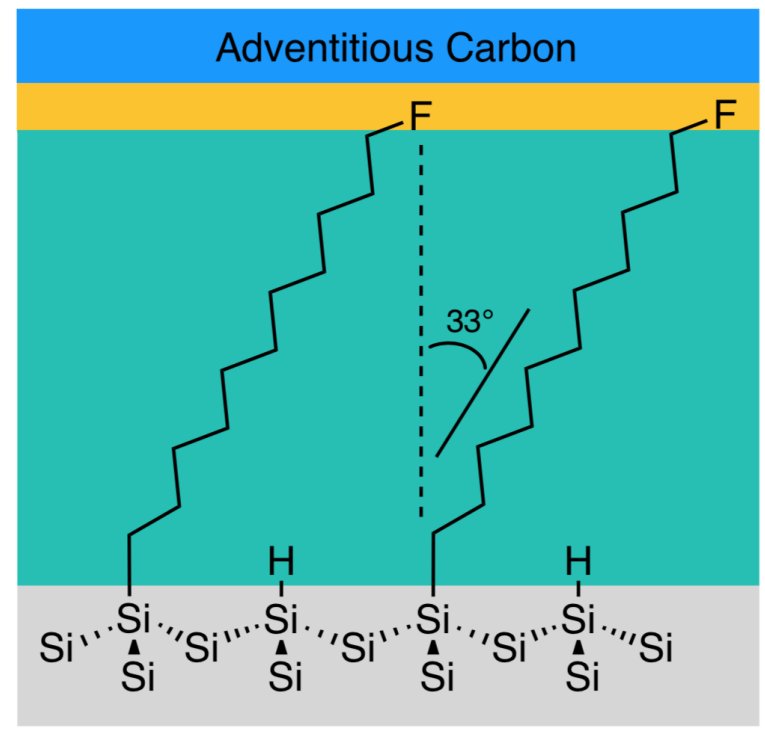

Figure 5. Scheme of the cross-section of the organic monolayer comprising $\equiv \mathrm{Si}-\left(\mathrm{CH}_{2}\right)_{11}-\mathrm{F}$ groups, an $\omega$-heteroatomic tag. 
Within the continuum model as shown in Figure 5, the total Si 2 p signal intensity at the alkyl chain-modified surface is given by

$$
\begin{gathered}
I_{\mathrm{Si}}=\sigma_{\mathrm{Si}} \rho_{\mathrm{Si}}\left(\int_{0}^{\infty} \exp \left(-x / \lambda_{\mathrm{SiSi}}\right) \mathrm{d} x\right) \exp \left(-d_{\mathrm{C}} / \lambda_{\mathrm{SiC}}\right) \exp \left(-d_{\mathrm{F}} / \lambda_{\mathrm{SiF}}\right) \exp \left(-d_{\mathrm{A}} / \lambda_{\mathrm{SiA}}\right) \\
I_{\mathrm{Si}}=\sigma_{\mathrm{Si}} \rho_{\mathrm{Si}} \lambda_{\mathrm{SiSi}} \exp \left(-d_{\mathrm{C}}^{\mathrm{Si}} / \lambda_{\mathrm{SiC}}-d_{\mathrm{F}} / \lambda_{\mathrm{SiF}}-d_{\mathrm{A}} / \lambda_{\mathrm{SiA}}\right)
\end{gathered}
$$

If we assume $d_{\mathrm{F}} / \lambda_{\mathrm{SiF}} \ll 1$, and $\lambda_{\mathrm{SiC}}^{\mathrm{Si}}=\frac{\rho_{\mathrm{C}}^{\mathrm{Au}}}{\rho_{\mathrm{C}}^{\mathrm{Si}}} \lambda_{\mathrm{SiC}}^{\mathrm{Au}}$, Eq 14 can be written as

$$
I_{\mathrm{Si}}=\sigma_{\mathrm{Si}} \rho_{\mathrm{Si}} \lambda_{\mathrm{SiSi}} \exp \left(\frac{-d_{\mathrm{C}}^{\mathrm{Si}} \rho_{\mathrm{C}}^{\mathrm{Si}}}{\rho_{\mathrm{C}}^{\mathrm{Au}} \lambda_{\mathrm{SiC}}^{\mathrm{Au}}}\right) \exp \left(-d_{\mathrm{A}} / \lambda_{\mathrm{SiA}}\right)
$$

Substituting Eq 10, $\rho_{C}^{S i}=\frac{\phi n_{S i} N_{\text {chain }}}{d_{C}^{S i}}$, Eq 15 becomes

$$
I_{\mathrm{Si}}=\sigma_{\mathrm{Si}} \rho_{\mathrm{Si}} \lambda_{\mathrm{SiSi}} \exp \left(-d_{\mathrm{A}} / \lambda_{\mathrm{SiA}}\right) \exp \left(\frac{-\phi n_{\mathrm{Si}} N_{\text {chain }}}{\rho_{\mathrm{C}}^{\mathrm{Au}} \lambda_{\mathrm{SiC}}^{\mathrm{Au}}}\right)
$$

The carbon signal from the alkyl group is given by

$$
\begin{gathered}
I_{\mathrm{C}}^{\mathrm{Si}}=\sigma_{\mathrm{C}}^{\mathrm{Si}} \rho_{\mathrm{C}}^{\mathrm{Si}}\left[\int_{0}^{d_{\mathrm{C}}^{\mathrm{Si}}} \exp \left(-x / \lambda_{\mathrm{CC}}\right) \mathrm{d} x\right] \exp \left(-d_{\mathrm{F}} / \lambda_{\mathrm{CF}}\right) \exp \left(-d_{\mathrm{A}} / \lambda_{\mathrm{CA}}\right) \\
I_{\mathrm{C}}^{\mathrm{Si}}=\sigma_{\mathrm{C}} \rho_{\mathrm{C}}^{\mathrm{Si}} \lambda_{\mathrm{CC}}^{\mathrm{Si}}\left[1-\exp \left(-d_{\mathrm{C}}^{\mathrm{Si}} / \lambda_{\mathrm{CC}}^{\mathrm{Si}}\right)\right] \exp \left(-d_{\mathrm{F}} / \lambda_{\mathrm{CF}}-d_{\mathrm{A}} / \lambda_{\mathrm{CA}}\right) \\
I_{\mathrm{C}}^{\mathrm{Si}}=\sigma_{\mathrm{C}} \rho_{\mathrm{C}}^{\mathrm{Au}} \lambda_{\mathrm{CC}}^{\mathrm{Au}}\left[1-\exp \left(\frac{-d_{\mathrm{C}}^{\mathrm{Si}} \rho_{\mathrm{C}}^{\mathrm{Si}}}{\rho_{\mathrm{C}}^{\mathrm{Au}} \lambda_{\mathrm{CC}}^{\mathrm{Au}}}\right)\right] \exp \left(-d_{\mathrm{A}} / \lambda_{\mathrm{CA}}\right) \\
I_{\mathrm{C}}^{\mathrm{Si}}=\sigma_{\mathrm{C}} \rho_{\mathrm{C}}^{\mathrm{Au}} \lambda_{\mathrm{CC}}^{\mathrm{Au}}\left[1-\exp \left(\frac{-\phi n_{\mathrm{Si}} N_{\mathrm{chain}}}{\rho_{\mathrm{C}}^{\mathrm{Au}} \lambda_{\mathrm{CC}}^{\mathrm{Au}}}\right)\right] \exp \left(-d_{\mathrm{A}} / \lambda_{\mathrm{CA}}\right)
\end{gathered}
$$

where the same assumptions and substitutions are made when deriving Eq 16. The signal from the fluorine layer is given by 


$$
\begin{gathered}
I_{\mathrm{F}}=\sigma_{\mathrm{F}} \rho_{\mathrm{F}}\left[\int_{0}^{d_{\mathrm{F}}} \exp \left(-x / \lambda_{\mathrm{FF}}\right) \mathrm{d} x\right] \exp \left(-d_{\mathrm{A}} / \lambda_{\mathrm{FA}}\right) \\
I_{\mathrm{F}}=\sigma_{\mathrm{F}} \rho_{\mathrm{F}} \lambda_{\mathrm{FF}}^{\mathrm{Si}}\left[1-\exp \left(-d_{\mathrm{F}} / \lambda_{\mathrm{FF}}^{\mathrm{Si}}\right)\right] \exp \left(-d_{\mathrm{A}} / \lambda_{\mathrm{FA}}\right)
\end{gathered}
$$

We can assume that $1-\exp \left(-d_{\mathrm{F}} / \lambda_{\mathrm{FF}}^{\mathrm{Si}}\right) \approx d_{\mathrm{F}} / \lambda_{\mathrm{FF}}^{\mathrm{Si}}, \mathrm{Eq} 18$ becomes

$$
I_{\mathrm{F}}=\sigma_{\mathrm{F}} \rho_{\mathrm{F}} \lambda_{\mathrm{FF}}^{\mathrm{Si}} \frac{d_{\mathrm{F}}}{\lambda_{\mathrm{FF}}^{\mathrm{Si}}} \exp \left(-d_{\mathrm{A}} / \lambda_{\mathrm{FA}}\right)=\sigma_{\mathrm{F}} \rho_{\mathrm{F}} d_{\mathrm{F}} \exp \left(-d_{\mathrm{A}} / \lambda_{\mathrm{FA}}\right)
$$

Given that $\rho_{\mathrm{F}}^{\mathrm{Si}}=\frac{\phi n_{\mathrm{Si}}}{d_{\mathrm{F}}}$, the signal from the fluorine layer is given by

$$
I_{\mathrm{F}}=\sigma_{\mathrm{F}} \phi n_{\mathrm{Si}} \exp \left(-d_{\mathrm{A}} / \lambda_{\mathrm{FA}}\right)
$$

The intensity of adventitious carbon signal is given by

$$
\begin{gathered}
I_{\mathrm{A}}=\sigma_{\mathrm{C}} \rho_{\mathrm{A}}\left[\int_{0}^{d_{\mathrm{A}}} \exp \left(-x / \lambda_{\mathrm{CA}}\right) \mathrm{dx}\right] \\
I_{\mathrm{A}}=\sigma_{\mathrm{C}} \rho_{\mathrm{A}}^{\mathrm{Au}} \lambda_{\mathrm{CC}}^{\mathrm{Au}}\left[1-\exp \left(-d_{\mathrm{A}} / \lambda_{\mathrm{CA}}\right)\right]
\end{gathered}
$$

Combining Eq 17 and 20, the total intensity of the carbon signal is given by

$$
I_{\mathrm{C}}^{\text {total }}=I_{\mathrm{C}}^{\mathrm{Si}}+I_{\mathrm{A}}=\sigma_{\mathrm{C}} \rho_{\mathrm{C}}^{\mathrm{Au}} \lambda_{\mathrm{CC}}^{\mathrm{Au}}\left[1-\exp \left(-d_{\mathrm{A}} / \lambda_{\mathrm{CA}}-\frac{\phi n_{\mathrm{Si}} N_{\text {chain }}}{\rho_{\mathrm{C}}^{\mathrm{Au}} \lambda_{\mathrm{CC}}^{\mathrm{Au}}}\right)\right]
$$

After obtaining the intensity of the silicon signal, $I_{\mathrm{Si}}(\mathrm{Eq} 16)$, the intensity of fluorine signal, $I_{\mathrm{F}}$ (Eq 19), and the total intensity of carbon signal, $I_{\mathrm{C}}{ }^{\text {total }}(\mathrm{Eq} 21)$, two quantification methods for the substitution level, using the ratio of $\mathrm{C}$ to $\mathrm{Si}, I_{\mathrm{C}}^{\text {total }} / I_{\mathrm{Si}}$, or the ratio of $\mathrm{F}$ to $\mathrm{Si}, I_{\mathrm{F}} / I_{\mathrm{Si}}$, respectively, will be derived as shown below. 
Substitution Level from the Ratio of C to Si. Dividing Eq 16 by Eq 21 gives the ratio of the total carbon intensity to the silicon intensity,

$$
\begin{gathered}
\frac{I_{\mathrm{C}}^{\text {total }}}{I_{\mathrm{Si}}}=\left(\frac{\sigma_{\mathrm{C}} \rho_{\mathrm{C}}^{\mathrm{Au}} \lambda_{\mathrm{CC}}^{\mathrm{Au}}}{\sigma_{\mathrm{Si}} \rho_{\mathrm{Si}} \lambda_{\mathrm{SiSi}}}\right)\left[\exp \left(\frac{d_{\mathrm{A}}}{\lambda_{\mathrm{SiA}}}+\frac{\phi n_{\mathrm{Si}} N_{\text {chain }}}{\rho_{\mathrm{C}}^{\mathrm{Au}} \lambda_{\mathrm{SiC}}^{\mathrm{Au}}}\right)\right. \\
\left.-\exp \left(\left(\frac{d_{\mathrm{A}}}{\lambda_{\mathrm{SiA}}}-\frac{d_{\mathrm{A}}}{\lambda_{\mathrm{CA}}}\right)+\frac{\phi n_{\mathrm{Si}} N_{\text {chain }}}{\rho_{\mathrm{C}}^{\mathrm{Au}}}\left(\frac{1}{\lambda_{\mathrm{SiC}}^{\mathrm{Au}}}-\frac{1}{\lambda_{\mathrm{CC}}^{\mathrm{Au}}}\right)\right)\right]
\end{gathered}
$$

Noting that $\frac{\phi n_{\mathrm{Si}} N_{\text {chain }}}{\rho_{\mathrm{C}}^{\mathrm{Au}}}\left(\frac{1}{\lambda_{\mathrm{SiC}}^{\mathrm{Au}}}-\frac{1}{\lambda_{\mathrm{CC}}^{\mathrm{Au}}}\right)=\phi N_{\text {chain }}(-0.00558)$ and $N_{\text {chain }} \leq 18$, we can derive the inequality

$$
0.9<\exp \left[\frac{\phi n_{\mathrm{Si}} N_{\text {chain }}}{\rho_{\mathrm{C}}^{\mathrm{Au}}}\left(\frac{1}{\lambda_{\mathrm{SiC}}^{\mathrm{Au}}}-\frac{1}{\lambda_{\mathrm{CC}}^{\mathrm{Au}}}\right)\right]<1
$$

Eq 22 becomes

$$
\begin{gathered}
\frac{I_{\mathrm{C}}^{\mathrm{total}}}{I_{\mathrm{Si}}} \approx\left(\frac{\sigma_{\mathrm{C}} \rho_{\mathrm{C}}^{\mathrm{Au}} \lambda_{\mathrm{CC}}^{\mathrm{Au}}}{\sigma_{\mathrm{Si}} \rho_{\mathrm{Si}} \lambda_{\mathrm{SiSi}}}\right)\left[\exp \left(\frac{d_{\mathrm{A}}}{\lambda_{\mathrm{SiA}}}+\frac{\phi n_{\mathrm{Si}} N_{\text {chain }}}{\rho_{\mathrm{C}}^{\mathrm{Au}} \lambda_{\mathrm{SiC}}^{\mathrm{Au}}}\right)-1\right] \\
\frac{d_{\mathrm{A}}}{\lambda_{\mathrm{SiA}}}+\frac{\phi n_{\mathrm{Si}} N_{\text {chain }}}{\rho_{\mathrm{C}}^{\mathrm{Au}} \lambda_{\mathrm{SiC}}^{\mathrm{Au}}}=\ln \left[\frac{I_{\mathrm{C}}^{\mathrm{total}}}{I_{\mathrm{Si}}}\left(\frac{\sigma_{\mathrm{Si}} \rho_{\mathrm{Si}} \lambda_{\mathrm{SiSi}}}{\sigma_{\mathrm{C}} \rho_{\mathrm{C}}^{\mathrm{Au}} \lambda_{\mathrm{CC}}^{\mathrm{Au}}}\right)+1\right]
\end{gathered}
$$

and $\varphi$ can be calculated as

$$
\begin{gathered}
\phi=\frac{\rho_{\mathrm{C}}^{\mathrm{Au}} \lambda_{\mathrm{SiC}}^{\mathrm{Au}}}{n_{\mathrm{Si}} N_{\text {chain }}} \ln \left[\frac{I_{\mathrm{C}}^{\mathrm{total}}}{I_{\mathrm{Si}}}\left(\frac{\sigma_{\mathrm{Si}} \rho_{\mathrm{Si}} \lambda_{\mathrm{SiSi}}}{\sigma_{\mathrm{C}} \rho_{\mathrm{C}}^{\mathrm{Au}} \lambda_{\mathrm{CC}}^{\mathrm{Au}}}\right)+1\right]-\frac{\rho_{\mathrm{C}}^{\mathrm{Au}} \lambda_{\mathrm{SiC}}^{\mathrm{Au}} d_{\mathrm{A}}}{n_{\mathrm{Si}} N_{\mathrm{chain}} \lambda_{\mathrm{SiA}}} \\
\phi=\frac{\rho_{\mathrm{C}}^{\mathrm{Au}} \lambda_{\mathrm{SiC}}^{\mathrm{Au}}}{n_{\mathrm{Si}} N_{\text {chain }}} \ln \left[\frac{I_{\mathrm{C}}^{\mathrm{total}}}{I_{\mathrm{Si}}}\left(\frac{\sigma_{\mathrm{Si}} \rho_{\mathrm{Si}} \lambda_{\mathrm{SiSi}}}{\sigma_{\mathrm{C}} \rho_{\mathrm{C}}^{\mathrm{Au}} \lambda_{\mathrm{CC}}^{\mathrm{Au}}}\right)+1\right]-\frac{\rho_{\mathrm{A}} d_{\mathrm{A}}}{n_{\mathrm{Si}} N_{\text {chain }}}
\end{gathered}
$$

Eq 23 shows the relation between the substitution level and related parameters, derived from the $\mathrm{C} / \mathrm{Si}$ ratio. If we assume $k$ is the ratio of the atomic density of adventitious carbon on silicon, 
$\rho_{\mathrm{A}}$, to the atomic density of carbon on gold, $\rho_{\mathrm{C}}^{\mathrm{Au}}$, in other words, $\rho_{\mathrm{A}}=k \rho_{\mathrm{C}}^{\mathrm{Au}}$, and put all the constants from Table 5 into Eq 23

$$
\begin{aligned}
& \phi=\frac{\left(4.1 \times 10^{22}\right)\left(3.95 \times 10^{-7}\right)}{\left(7.8 \times 10^{14}\right) N_{\text {chain }}} \ln \left[\frac{I_{\mathrm{C}}^{\text {total }}}{I_{\mathrm{Si}}}(0.732)+1\right]-\frac{k \rho_{\mathrm{C}}^{\mathrm{Au}} d_{\mathrm{A}}(\mathrm{nm}) \times 10^{-7}}{\left(7.8 \times 10^{14}\right) N_{\text {chain }}} \\
& \phi=\frac{20.76}{N_{\text {chain }}} \ln \left(0.732 \frac{I_{\mathrm{C}}^{\text {total }}}{I_{\mathrm{Si}}}+1\right)-5.26 \frac{k d_{\mathrm{A}}}{N_{\text {chain }}}
\end{aligned}
$$

If we assume $k=0.8, N_{\text {chain }}=11$ for the $\equiv \mathrm{Si}-\left(\mathrm{CH}_{2}\right)_{11}-\mathrm{F}$ surface, and $I_{\mathrm{C}}^{\mathrm{total}} / I_{\mathrm{Si}}=0.441$ (Table 4$)$, Eq 24 becomes

$$
\phi=0.527-0.38 d_{\mathrm{A}}
$$

$\mathrm{Eq} 23$ is a general formula that uses the $\mathrm{C} / \mathrm{Si}$ ratio for the quantification of the substitution level of Si-H groups by alkyl chains on the silicon surface. After we consider all the constants and the actual $\mathrm{C} / \mathrm{Si}$ ratio from XPS data, and assume the ratio of atom density of adventitious carbon on silicon to that of carbon on gold has a value of 0.8 , the obtained Eq 25 shows the relationship between the substitution level and the thickness of the adventitious carbon. From Eq 25, we can see the coefficient before the thickness is 0.38 , which means the change of the thickness of adventitious carbon will result in a significant change of the value of substitution level if the $\mathrm{C} / \mathrm{Si}$ ratio is used to quantify it. However, if the functional group on the silicon surface contains heteroatom(s) on the chain, another formula using the ratio of the heteroatom to silicon can be derived for the quantification of substitution levels (vide infra).

Determination of Substitution Levels from the Ratio of $\mathbf{F}$ to Si. Since the $\equiv \mathrm{Si}-\left(\mathrm{CH}_{2}\right)_{11}-\mathrm{F}$ surface contains another element, fluorine, the substitution level also can be obtained using the $\mathrm{F} / \mathrm{Si}$ ratio. Taking the ratio of fluorine to silicon intensities gives (Eqs 19 and 16) 


$$
\begin{gathered}
\frac{I_{\mathrm{F}}}{I_{\mathrm{Si}}}=\frac{\sigma_{\mathrm{F}} \phi n_{\mathrm{Si}} \exp \left(-d_{\mathrm{A}} / \lambda_{\mathrm{FA}}\right)}{\sigma_{\mathrm{Si}} \rho_{\mathrm{Si}} \lambda_{\mathrm{SiSi}} \exp \left(-d_{\mathrm{A}} / \lambda_{\mathrm{SiA}}\right) \exp \left(\frac{-\phi n_{S_{\mathrm{Si}} N_{\text {chain }}}}{\rho_{\mathrm{C}}^{\mathrm{Au}} \lambda_{\mathrm{SiC}}^{\mathrm{Ai}}}\right)} \\
\frac{I_{\mathrm{F}}}{I_{\mathrm{Si}}} \frac{\sigma_{\mathrm{Si}} \rho_{\mathrm{Si}} \lambda_{\mathrm{SiSi}}}{\sigma_{\mathrm{F}} n_{\mathrm{Si}}} \exp \left[d_{\mathrm{A}}\left(\frac{1}{\lambda_{\mathrm{FA}}}-\frac{1}{\lambda_{\mathrm{SiA}}}\right)\right]=\phi \exp \left(\frac{n_{\mathrm{Si}} N_{\text {chain }}}{\left.\rho_{\mathrm{C}}^{\mathrm{Au}} \lambda_{\mathrm{SiC}}^{\mathrm{Au}} \phi\right)}\right.
\end{gathered}
$$

If we first-order Taylor expand

$$
\exp \left(\frac{n_{\mathrm{Si}} N_{\text {chain }}}{\rho_{\mathrm{C}}^{\mathrm{Au}} \lambda_{\mathrm{SiC}}^{\mathrm{Au}}} \phi\right) \approx 1+\frac{n_{\mathrm{Si}} N_{\text {chain }}}{\rho_{\mathrm{C}}^{\mathrm{Au}} \lambda_{\mathrm{SiC}}^{\mathrm{Au}}} \phi
$$

Eq 26 becomes

$$
\frac{I_{\mathrm{F}}}{I_{\mathrm{Si}}} \frac{\sigma_{\mathrm{Si}} \rho_{\mathrm{Si}} \lambda_{\mathrm{SiSi}}}{\sigma_{\mathrm{F}} n_{\mathrm{Si}}} \exp \left[d_{\mathrm{A}}\left(\frac{1}{\lambda_{\mathrm{FA}}}-\frac{1}{\lambda_{\mathrm{SiA}}}\right)\right]=\phi\left(1+\frac{n_{\mathrm{Si}} N_{\text {chain }}}{\rho_{\mathrm{C}}^{\mathrm{Au}} \lambda_{\mathrm{SiC}}^{\mathrm{Au}}} \phi\right)
$$

Eq 27 shows the relationship between the substitution level and all the related parameters, derived from the F/Si ratio. As mentioned, $\lambda_{\mathrm{FA}} \rho_{\mathrm{A}}=\lambda_{\mathrm{FC}}^{\mathrm{Au}} \rho_{\mathrm{C}}^{\mathrm{Au}}, \lambda_{\mathrm{SiA}} \rho_{\mathrm{A}}=\lambda_{\mathrm{SiC}}^{\mathrm{Au}} \rho_{\mathrm{C}}^{\mathrm{Au}}, \rho_{\mathrm{A}}=k \rho_{\mathrm{C}}^{\mathrm{Au}}$, and we assume $k=0.8$. If we put all the constants from Table 5 into Eq 27,

$$
\begin{gathered}
\frac{I_{\mathrm{F}}}{I_{\mathrm{Si}}} \frac{(0.328)\left(5 \times 10^{22}\right)\left(1.8 \times 10^{-7}\right)}{(1)\left(7.8 \times 10^{14}\right)} \exp \left[d_{A}(0.8)\left(\frac{1}{2.66}-\frac{1}{3.95}\right)\right]=\phi\left[1+\frac{\left(7.8 \times 10^{14}\right)(11)}{\left(4.1 \times 10^{22}\right)\left(3.95 \times 10^{-7}\right)} \phi\right] \\
\frac{I_{\mathrm{F}}}{I_{\mathrm{Si}}}(3.78) \exp \left(0.1 d_{\mathrm{A}}\right)=\phi(1+0.53 \phi)
\end{gathered}
$$

We can solve the quadratic Eq 28 for $\varphi$

$$
\phi=\frac{\sqrt{1+8 \frac{I_{\mathrm{F}}}{I_{\mathrm{Si}}} \exp \left(0.1 d_{\mathrm{A}}\right)}-1}{1.06}
$$


If we first-order Taylor expand $\exp \left(0.1 d_{\mathrm{A}}\right) \approx 1+0.1 d_{\mathrm{A}}$, and designate the fluorine to silicon intensity ratio of 0.187 from the XPS data from Table 4 into Eq 29, then, Eq 29 becomes

$$
\phi=\frac{\sqrt{2.5+0.15 d_{\mathrm{A}}}-1}{1.06}
$$

Taylor expanding the square root of Eq 30 gives

$$
\phi=0.547+0.045 d_{\mathrm{A}}
$$

Eq 27 is a general formula that uses the $\mathrm{F} / \mathrm{Si}$ ratio for the quantification of the substitution level of Si-H groups by alkyl chains on the silicon surface. After we consider all the constants, the actual F/Si ratio from XPS data, and make reasonable assumptions, the obtained Eq 31 shows the relationship between the substitution level and the thickness of the adventitious carbon. The coefficient before the value of thickness is 0.045 , which reflects the much smaller influence of adventitious carbon on the calculated values of substitution levels if one uses the F/Si ratio (or ratio of any heteroatomic label), for quantification.

It is worth noting that our final expression differs from that of Yamazaki et al., ${ }^{35}$ in that they assumed the attenuation length was independent of the substitution level. If we assume no adventitious carbon on the surface and that the attenuation length is independent of the substitution level of the monolayer, the ratio of fluorine to silicon intensities (Eq 19 and Eq 14) gives Eq 32, which converges with Eq 3 from the work of Yamazaki et al.

$$
\begin{gathered}
\frac{I_{\mathrm{F}}}{I_{\mathrm{Si}}}=\frac{\sigma_{\mathrm{F}} \phi n_{\mathrm{Si}}}{\sigma_{\mathrm{Si}} \rho_{\mathrm{Si}} \lambda_{\mathrm{SiSi}} \exp \left(-d_{\mathrm{C}} / \lambda_{\mathrm{SiC}}-d_{\mathrm{F}} / \lambda_{\mathrm{SiF}}\right)} \\
=\frac{\sigma_{\mathrm{F}} \phi d_{\mathrm{Si}}}{\sigma_{\mathrm{Si}} \lambda_{\mathrm{SiSi}} \exp \left(-d_{\mathrm{ml}} / \lambda_{\mathrm{ml}}\right)} \\
=\frac{\sigma_{\mathrm{F}}}{\sigma_{\mathrm{Si}}} \phi \frac{d_{\mathrm{Si}} / \lambda_{\mathrm{SiSi}}}{\exp \left(-d_{\mathrm{ml}} / \lambda_{\mathrm{ml}}\right)} \\
\approx \frac{\sigma_{\mathrm{F}}}{\sigma_{\mathrm{Si}}} \phi \frac{\left[1-\exp \left(-d_{\mathrm{Si}} / \lambda_{\mathrm{SiSi}}\right)\right]}{\exp \left(-d_{\mathrm{ml}} / \lambda_{\mathrm{ml}}\right)}
\end{gathered}
$$




$$
\phi=\frac{I_{\mathrm{F}}}{I_{\mathrm{Si}}} \frac{\sigma_{\mathrm{Si}}}{\sigma_{\mathrm{F}}} \frac{\exp \left(-d_{\mathrm{ml}} / \lambda_{\mathrm{ml}}\right)}{\left[1-\exp \left(-d_{\mathrm{Si}} / \lambda_{\mathrm{SiSi}}\right)\right]}
$$

Monolayers without heteroatomic tags: $\equiv \mathrm{Si}-\left(\mathrm{CH}_{2}\right)_{n}-\mathrm{CH}_{3}$ surfaces.

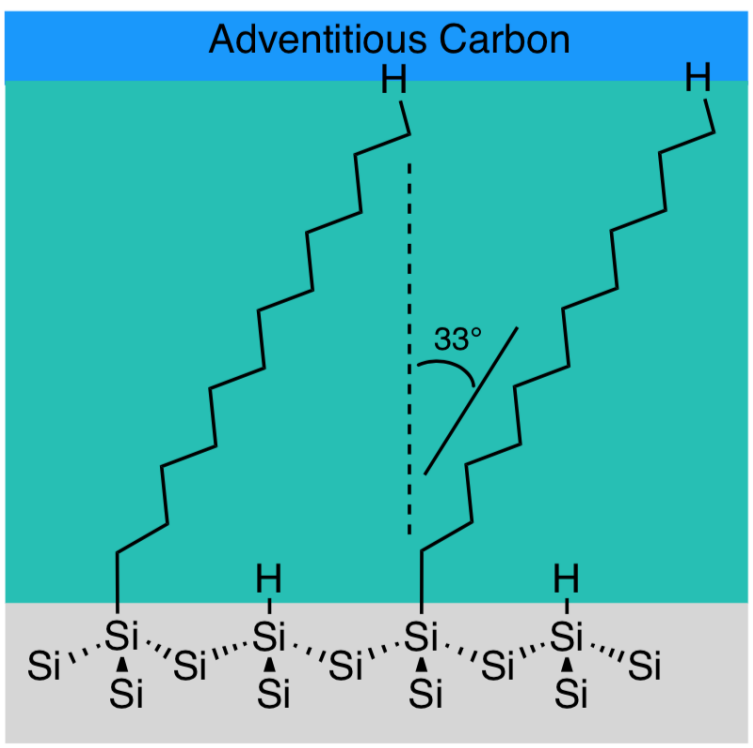

Figure 6. Scheme of the cross-section of the organic monolayer comprising $\equiv \mathrm{Si}-\left(\mathrm{CH}_{2}\right)_{n}-\mathrm{CH}_{3}$ groups.

A three layer model is used for the $\equiv \mathrm{Si}-\left(\mathrm{CH}_{2}\right)_{n}-\mathrm{CH}_{3}$ surfaces, including the silicon layer at the bottom, an organic alkyl monolayer, and an adventitious carbon layer, as shown in Figure 6. Similarly, the signal from silicon layer is given by

$$
\begin{aligned}
& I_{\mathrm{Si}}=\sigma_{\mathrm{Si}} \rho_{\mathrm{Si}}\left[\int_{0}^{\infty} \exp \left(-x / \lambda_{\mathrm{SiSi}}\right) \mathrm{d} x\right] \exp \left(-d_{\mathrm{C}} / \lambda_{\mathrm{SiC}}\right) \exp \left(-d_{\mathrm{A}} / \lambda_{\mathrm{SiA}}\right) \\
& I_{\mathrm{Si}}=\sigma_{\mathrm{Si}} \rho_{\mathrm{Si}} \lambda_{\mathrm{SiSi}} \exp \left(-d_{\mathrm{C}} / \lambda_{\mathrm{SiC}}-d_{\mathrm{A}} / \lambda_{\mathrm{SiA}}\right)
\end{aligned}
$$

The carbon signal from the alkyl group is given by

$$
I_{\mathrm{C}}^{\mathrm{Si}}=A \sigma_{\mathrm{C}} \rho_{\mathrm{C}} \lambda_{\mathrm{CC}}\left[1-\exp \left(-d_{\mathrm{C}} / \lambda_{\mathrm{CC}}\right)\right] \exp \left(-d_{\mathrm{A}} / \lambda_{\mathrm{CA}}\right)
$$


The signal of adventitious carbon is given by

$$
I_{\mathrm{A}}=\sigma_{\mathrm{C}} \rho_{\mathrm{A}} \lambda_{\mathrm{CA}}\left[1-\exp \left(-d_{\mathrm{A}} / \lambda_{\mathrm{CA}}\right)\right]
$$

Similar to the $\equiv \mathrm{Si}-\left(\mathrm{CH}_{2}\right)_{11}-\mathrm{F}$ surface, the total intensity of carbon signal is

$$
I_{\mathrm{C}}^{\text {total }}=\sigma_{\mathrm{C}} \rho_{\mathrm{C}}^{\mathrm{Au}} \lambda_{\mathrm{CC}}^{\mathrm{Au}}\left[1-\exp \left(-d_{\mathrm{A}} / \lambda_{\mathrm{CA}}-\frac{\phi n_{\mathrm{Si}} N_{\text {chain }}}{\rho_{\mathrm{C}}^{\mathrm{Au}} \lambda_{\mathrm{CC}}^{\mathrm{Au}}}\right)\right]
$$

Substitution Level from the Ratio of C to Si. Taking the ratio of $I_{\mathrm{C}}{ }^{\text {total }} / I_{\mathrm{Si}}$ also yields the same equation as Eq 23

$$
\phi=\frac{\rho_{\mathrm{C}}^{\mathrm{Au}} \lambda_{\mathrm{SiC}}^{\mathrm{Au}}}{n_{\mathrm{Si}} N_{\text {chain }}} \ln \left[\frac{I_{\mathrm{C}}^{\mathrm{total}}}{I_{\mathrm{Si}}}\left(\frac{\sigma_{\mathrm{Si}} \rho_{\mathrm{Si}} \lambda_{\mathrm{SiSi}}}{\sigma_{\mathrm{C}} \rho_{\mathrm{C}}^{\mathrm{Au}} \lambda_{\mathrm{CC}}^{\mathrm{Au}}}\right)+1\right]-\frac{\rho_{\mathrm{A}} d_{\mathrm{A}}}{n_{\mathrm{Si}} N_{\text {chain }}}
$$

Similar to the $\equiv \mathrm{Si}-\left(\mathrm{CH}_{2}\right)_{11}-\mathrm{F}$ surface, after putting all the constants into the Eq 22, the coverage is given by

$$
\phi=\frac{20.76}{N_{\text {chain }}} \ln \left(0.732 \frac{I_{\mathrm{C}}^{\text {total }}}{I_{\mathrm{Si}}}+1\right)-5.26 \frac{k d_{\mathrm{A}}}{N_{\text {chain }}}
$$

For the $\equiv \mathrm{Si}-\left(\mathrm{CH}_{2}\right)_{7}-\mathrm{CH}_{3}$ surface, the carbon to silicon ratio obtained from XPS is 0.362 (Table 4 ), $N_{\text {chain }}=8$, and if we again assume $k=0.8$, then the coverage is given by

$$
\phi=0.61-0.53 d_{\mathrm{A}}
$$

For the $\equiv \mathrm{Si}-\left(\mathrm{CH}_{2}\right)_{11}-\mathrm{CH}_{3}$ surface, the carbon to silicon ratio obtained from XPS is 0.523 (Table 4 ), $N_{\text {chain }}=12$, and if we again assume $k=0.8$, then the coverage is given by

$$
\phi=0.56-0.35 d_{\mathrm{A}}
$$

For the $\equiv \mathrm{Si}-\left(\mathrm{CH}_{2}\right)_{17}-\mathrm{CH}_{3}$ surface, the carbon to silicon ratio obtained from XPS is 0.790 (Table 4 ), $N_{\text {chain }}=18$, and if we again assume $k=0.8$, then the coverage is given by 


$$
\phi=0.53-0.23 d_{\mathrm{A}}
$$

Eqs 36a, $b$, and $c$ reveal the relationships of substitution levels with the thicknesses of the adventitious carbon on the pure alkyl-terminated silicon surfaces. The coefficients of the thickness in the formulae for the pure alkyl terminated silicon surfaces are $0.53,0.35$, and 0.23 , for the $\equiv \mathrm{Si}-\left(\mathrm{CH}_{2}\right)_{7}-\mathrm{CH}_{3}, \equiv \mathrm{Si}-\left(\mathrm{CH}_{2}\right)_{11}-\mathrm{CH}_{3}$, and $\equiv \mathrm{Si}-\left(\mathrm{CH}_{2}\right)_{17}-\mathrm{CH}_{3}$ surfaces, respectively. Similarly to the formula using the $\mathrm{C} / \mathrm{Si}$ ratio for the fluorine-terminated silicon surface, the small change of the thickness of adventitious or physisorbed hydrocarbons on silicon surface will have a significant effect on the calculated values of substitution levels.

\section{Monolayers with an $\alpha$-heteroatom, surface-bound tag: the $\equiv \mathrm{Si}-\mathrm{S}-\left(\mathrm{CH}_{2}\right)_{17}-\mathrm{CH}_{3}$} surface. In our previous work, we derived two expressions for the substitution level, using the ratio of carbon to silicon or the ratio of sulfur to silicon. ${ }^{43}$ The scheme of the layered model is shown in Figure 7. Here, an updated formula for the $\equiv \mathrm{Si}-\mathrm{S}-\left(\mathrm{CH}_{2}\right)_{17}-\mathrm{CH}_{3}$ surface is derived.

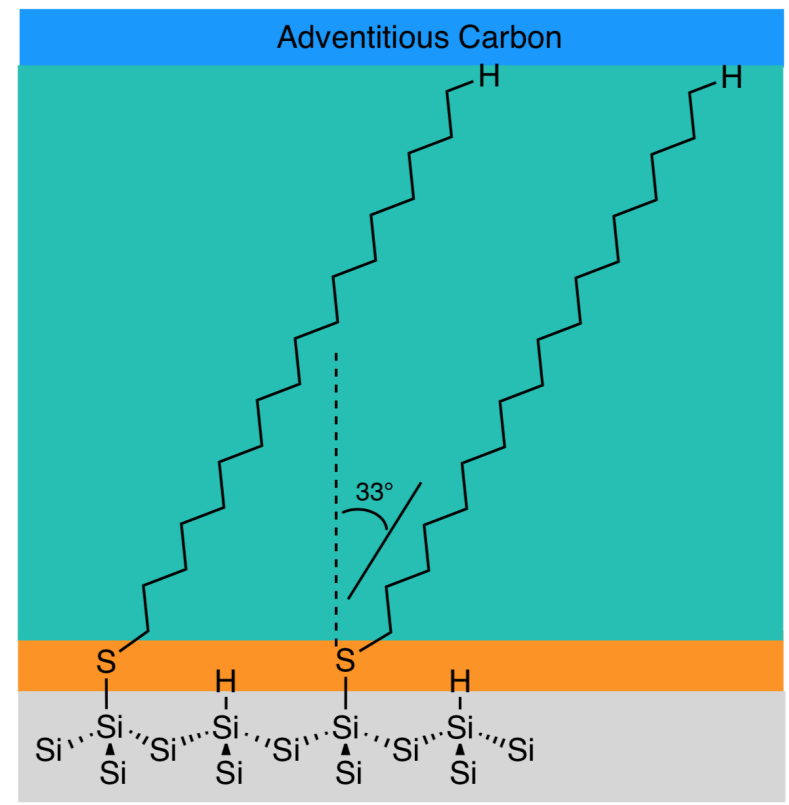

Figure 7. Scheme of the cross-section of the organic monolayer comprising $\equiv \mathrm{Si}-\mathrm{E}-\left(\mathrm{CH}_{2}\right)_{17}-\mathrm{CH}_{3}$ groups.

The signal of silicon is given by 


$$
I_{\mathrm{Si}}=\sigma_{\mathrm{Si}} \rho_{\mathrm{Si}}\left[\int_{0}^{\infty} \exp \left(-x / \lambda_{\mathrm{SiSi}}\right) \mathrm{d} x\right] \exp \left(-d_{\mathrm{E}} / \lambda_{\mathrm{SiE}}\right) \exp \left(-d_{\mathrm{C}} / \lambda_{\mathrm{SiC}}\right) \exp \left(-d_{\mathrm{A}} / \lambda_{\mathrm{SiA}}\right)
$$

If we assume $d_{E} / \lambda_{\operatorname{SiE}} \ll 1$,

$$
I_{\mathrm{Si}}=\sigma_{\mathrm{Si}} \rho_{\mathrm{Si}} \lambda_{\mathrm{SiSi}} \exp \left(-d_{\mathrm{C}} / \lambda_{\mathrm{SiC}}-d_{\mathrm{A}} / \lambda_{\mathrm{SiA}}\right)
$$

The signal of sulfur atom is given by

$$
\begin{gathered}
I_{\mathrm{S}}=\sigma_{\mathrm{S}} \rho_{\mathrm{S}}\left[\int_{0}^{d_{\mathrm{S}}} \exp \left(-x / \lambda_{\mathrm{SS}}\right) \mathrm{d} x\right] \exp \left(-d_{\mathrm{C}} / \lambda_{\mathrm{SC}}\right) \exp \left(-d_{\mathrm{A}} / \lambda_{\mathrm{SA}}\right) \\
I_{\mathrm{S}}=\sigma_{\mathrm{S}} \rho_{\mathrm{S}} \lambda_{\mathrm{SS}}\left[1-\exp \left(-d_{\mathrm{S}} / \lambda_{\mathrm{SS}}\right)\right] \exp \left(-d_{\mathrm{C}} / \lambda_{\mathrm{SC}}\right) \exp \left(-d_{\mathrm{A}} / \lambda_{\mathrm{SA}}\right)
\end{gathered}
$$

The carbon signal from the alkyl group is given by

$$
I_{\mathrm{C}}^{\mathrm{Si}}=A \sigma_{\mathrm{C}} \rho_{\mathrm{C}} \lambda_{\mathrm{CC}}\left[1-\exp \left(-d_{\mathrm{C}} / \lambda_{\mathrm{CC}}\right)\right] \exp \left(-d_{\mathrm{A}} / \lambda_{\mathrm{CA}}\right)
$$

The signal of adventitious carbon is given by

$$
I_{\mathrm{A}}=\sigma_{\mathrm{C}} \rho_{\mathrm{A}} \lambda_{\mathrm{CA}}\left[1-\exp \left(-d_{\mathrm{A}} / \lambda_{\mathrm{CA}}\right)\right]
$$

Substitution Level from the Ratio of $\mathbf{C}$ to $\mathrm{Si}$. Similar to the $\equiv \mathrm{Si}-\left(\mathrm{CH}_{2}\right)_{11}-\mathrm{F}$ surface, the substitution level is finally given by

$$
\phi=\frac{\rho_{\mathrm{C}}^{\mathrm{Au}} \lambda_{\mathrm{SiC}}^{\mathrm{Au}}}{n_{\mathrm{Si}} N_{\text {chain }}} \ln \left[\frac{I_{\mathrm{C}}^{\mathrm{total}}}{I_{\mathrm{Si}}}\left(\frac{\sigma_{\mathrm{Si}} \rho_{\mathrm{Si}} \lambda_{\mathrm{SiSi}}}{\sigma_{\mathrm{C}} \rho_{\mathrm{C}}^{\mathrm{Au}} \lambda_{\mathrm{CC}}^{\mathrm{Au}}}\right)+1\right]-\frac{\rho_{\mathrm{A}} d_{\mathrm{A}}}{n_{\mathrm{Si}} N_{\text {chain }}}
$$

and after putting all the constants into the Eq 23 , the coverage is given by

$$
\phi=\frac{20.76}{N_{\text {chain }}} \ln \left(0.732 \frac{I_{\mathrm{C}}^{\text {total }}}{I_{\mathrm{Si}}}+1\right)-5.26 \frac{k d_{\mathrm{A}}}{N_{\text {chain }}}
$$

For $\equiv \mathrm{Si}-\mathrm{S}-\left(\mathrm{CH}_{2}\right)_{17}-\mathrm{CH}_{3}$ surface, the carbon to silicon ratio is 0.749 (Table 4$), N_{\text {chain }}=18$, and $k=0.8$, and Eq 24 becomes 


$$
\phi=0.50-0.23 d_{\mathrm{A}}
$$

\section{Determination of the Substitution Level from the Ratio of S, a Surface-bound Atomic Tag,} to $\mathrm{Si}$. Since the $\equiv \mathrm{Si}-\mathrm{S}-\left(\mathrm{CH}_{2}\right)_{17}-\mathrm{CH}_{3}$ surface contains a distinct element, sulfur, bound to the

silicon surface, the substitution level also can be obtained using the S/Si ratio. Taking the ratio of sulfur (Eq 38) to silicon (Eq 37) intensities gives

$$
\frac{I_{\mathrm{S}}}{I_{\mathrm{Si}}}=\frac{\sigma_{\mathrm{E}} \rho_{\mathrm{S}} \lambda_{\mathrm{SS}}\left[1-\exp \left(-d_{\mathrm{S}} / \lambda_{\mathrm{SS}}\right)\right] \exp \left(-d_{\mathrm{C}} / \lambda_{\mathrm{SC}}\right) \exp \left(-d_{\mathrm{A}} / \lambda_{\mathrm{SA}}\right)}{\sigma_{\mathrm{Si}} \rho_{\mathrm{Si}} \lambda_{\mathrm{SiSi}} \exp \left(-d_{\mathrm{C}} / \lambda_{\mathrm{SiC}}-d_{\mathrm{A}} / \lambda_{\mathrm{SiA}}\right)}
$$

If we again assume that $\exp \left[d_{\mathrm{C}}\left(1 / \lambda_{\mathrm{SiC}}-1 / \lambda_{\mathrm{SC}}\right)\right] \approx 1, \exp \left[d_{\mathrm{A}}\left(1 / \lambda_{\mathrm{SiA}}-1 / \lambda_{\mathrm{SA}}\right)\right] \approx 1$, and $\mathrm{d}_{\mathrm{S}} / \lambda_{\mathrm{SS}} \ll 1$, we arrive at the simplified expression of

$$
\frac{I_{\mathrm{S}}}{I_{\mathrm{Si}}}=\frac{\sigma_{\mathrm{S}} \rho_{\mathrm{S}} d_{\mathrm{S}}}{\sigma_{\mathrm{Si}} \rho_{\mathrm{Si}} \lambda_{\mathrm{SiSi}}}
$$

Given that $\rho_{\mathrm{S}} d_{\mathrm{S}}=n_{\mathrm{S}}$, where $n_{\mathrm{S}}$ is the atomic surface density of sulfur atoms, and the substitution level is defined as $\phi=n_{\mathrm{S}} / n_{\mathrm{Si}}$, we can solve for the substitution level

$$
\phi=\frac{\sigma_{\mathrm{Si}} \rho_{\mathrm{Si}} \lambda_{\mathrm{SiSi}} I_{\mathrm{S}}}{n_{\mathrm{Si}} \sigma_{\mathrm{S}} I_{\mathrm{Si}}}
$$

After inputting the constants from Table 5, and $\mathrm{S} / \mathrm{Si}$ ratio of 0.052 into Eq 44, the substitution level determined was found to be $27 \%$. 


\section{Generalization of equations to non-alkyl hydrocarbon substitution and $\mathrm{Si}(100)$ (and other crystal faces)}

All of the above equations were derived under the assumption that the functional group covalently attached to the silicon surface was a linear alkyl chain (with or without a heteroatom). In the case of linear alkyl chains being grafted to the silicon surface, the areal atomic density of carbon atoms is uniform through the layer thickness perpendicular to the substrate. For more structurally complex molecules (e.g. aromatics or branched alkanes), it is still possible to apply the above formulas directly, where we simply need to modify Eq 10,

$$
\rho_{\mathrm{C}}^{\mathrm{Si}} \cong<\rho_{\mathrm{C}}^{\mathrm{Si}}>=\phi n_{\mathrm{Si}} N_{\mathrm{T}} / d_{\mathrm{C}}^{\mathrm{Si}}
$$

where the only change is to replace $N_{\text {Chain }}$ with $N_{\mathrm{T}}$, where $N_{\mathrm{T}}$, is the total number of carbon atoms in a single molecule being grafted to the surface and $d_{\mathrm{C}}^{\mathrm{Si}}$ is the height, relative to the substrate normal, of the molecule in question. This modification to Eq 10 is making the mean field assumption that the average atomic density of carbon atoms can be used to approximate the electron attenuation through the molecularly grafted layer. This is likely a fine approximation for most molecules, as there is unlikely to be large spatial fluctuations in density. In the case of a long linear chain with a bulky head group, it may be necessary to divide the carbon layer into two separate carbon layers with different densities and thicknesses.

It is also worth noting that all of the above simplified equations were derived assuming a $\mathrm{Si}(111)$ substrate. However, the equations are completely generalized to any orientation of the silicon surface, all that needs to be known is the atomic density of silicon surface atoms $n_{\mathrm{Si}}$, for the orientation of silicon used. Given that in all equations the substitution level on a $h k l$ oriented $\mathrm{Si}$ surface $\phi_{h k l}$, is inversely proportional to the atomic surface density of silicon atoms $n_{\mathrm{Si}}^{h k l}$, the substitution level on a $h^{\prime} k^{\prime} l^{\prime}$ oriented surface would be

$$
\phi_{h k^{\prime} l l^{\prime}}=\frac{n_{S i}^{h k l}}{n_{S i}^{h k^{\prime} l^{\prime}}} \phi_{h k l}
$$

For example, if the surface density of silicon atoms is taken to be $6.8 \times 10^{14}$ atoms $/ \mathrm{cm}^{2}$, this means that all of the above simplified equations for substitution level (e.g. equations 24, 25, 29, 
30, 31, 36a-c, 41 and 44) can be modified for a $\mathrm{Si}(100)$ surface by simply multiplying by a factor of $7.8 / 6.8=1.15$.

\section{Discussion}

The formulae derived for the quantification of substitution levels for the three types of functionalized silicon surfaces, as derived vide supra, are summarized in Table 6. According to the formulae using the $\mathrm{C} / \mathrm{Si}$ ratio, assuming no adventitious carbon on the surfaces, the substitution levels of the five surfaces are all in the 50-60\% range, in good agreement with those from the literature.

Table 6. Quantification of Substitution Level for Different Functionalized Si(111)-H Surfaces

\begin{tabular}{lccc}
\hline Surface & \multicolumn{1}{c}{${\mathrm{C} \text { to } \mathrm{Si}^{a}}^{a}$} & $\mathrm{~F}$ to $\mathrm{Si}^{a}$ & $\mathrm{~S}^{a} \mathrm{Si}^{a}$ \\
\hline$\equiv \mathrm{Si}-\left(\mathrm{CH}_{2}\right)_{11}-\mathrm{F}$ & $\phi=0.527-0.38 d_{\mathrm{A}}$ & $\phi=0.547+0.045 d_{\mathrm{A}}$ & -- \\
$\equiv \mathrm{Si}-\left(\mathrm{CH}_{2}\right)_{7}-\mathrm{CH}_{3}$ & $\phi=0.61-0.53 d_{\mathrm{A}}$ & -- & - \\
$\equiv \mathrm{Si}-\left(\mathrm{CH}_{2}\right)_{11}-\mathrm{CH}_{3}$ & $\phi=0.56-0.35 d_{\mathrm{A}}$ & -- & - \\
$\equiv \mathrm{Si}-\left(\mathrm{CH}_{2}\right)_{17}-\mathrm{CH}_{3}$ & $\phi=0.53-0.23 d_{\mathrm{A}}$ & -- & - \\
$\equiv \mathrm{Si}-\mathrm{S}-\left(\mathrm{CH}_{2}\right)_{17}-\mathrm{CH}_{3}$ & $\phi=0.50-0.23 d_{\mathrm{A}}$ & -- & $\phi=27 \%$ \\
\hline${ }^{a} \phi$ is the substution level and $d_{\mathrm{A}}$ is the thickness of adventitious carbon layer.
\end{tabular}

(a)

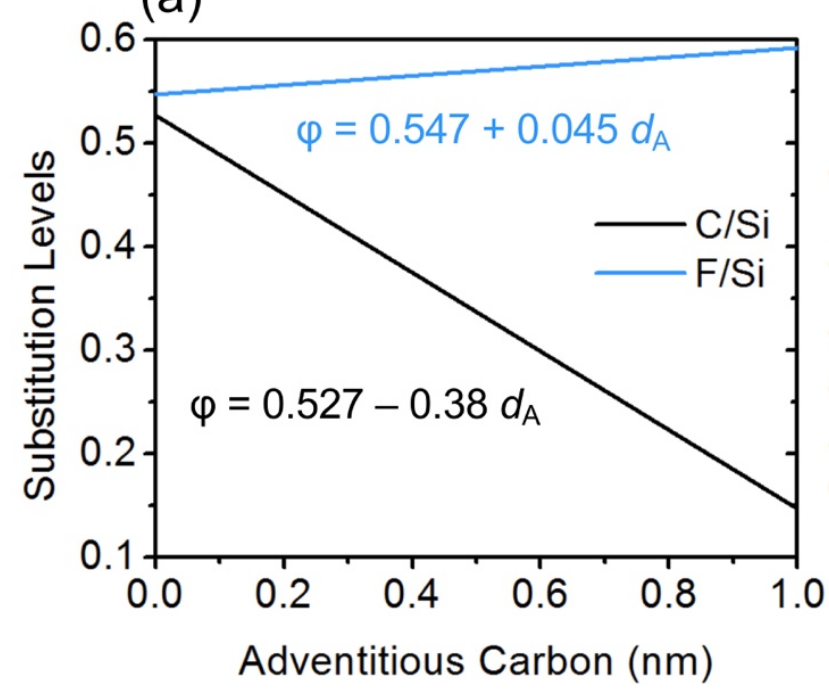

(b)

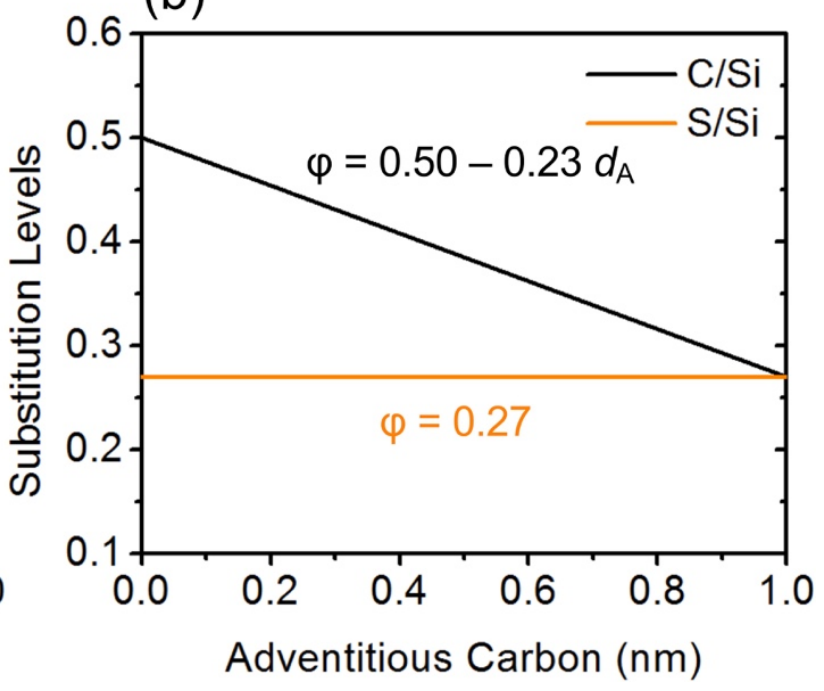

Figure 8. Scheme showing the relationship between substitution levels and the thickness of any possible adventitious hydrocarbon of (a) the $\equiv \mathrm{Si}(111)-\left(\mathrm{CH}_{2}\right)_{11}-\mathrm{F}$ surface, derived using the $\mathrm{C} / \mathrm{Si}$ ratio (black) or the $\mathrm{F} / \mathrm{Si}$ ratio (blue) from XPS data, and (b) the $\equiv \mathrm{Si}(111)-\mathrm{S}-\left(\mathrm{CH}_{2}\right)_{17}-\mathrm{CH}_{3}$ 
surface, derived using the $\mathrm{C} / \mathrm{Si}$ ratio (black) or the $\mathrm{S} / \mathrm{Si}$ ratio (orange) from XPS data. The data from this figure are tabulated in Table 6.

For the $\equiv \mathrm{Si}-\left(\mathrm{CH}_{2}\right)_{11}-\mathrm{F}$ surface, we then compared the results of the two derived equations (Table 6) to calculate substitution levels - one is based upon the C/Si ratio, and the other on the $\mathrm{F} / \mathrm{Si}$ ratio. When these two equations are plotted, as shown Figure 8a, the effect of adventitious hydrocarbon on the resulting XPS analysis can be seen to diverge in opposite directions; the substitution levels only agree when the thickness of the adventitious carbon layer is zero at a substitution level of $\sim 53 \%\left(d_{\mathrm{A}}=0\right)$. The effect of adventitious hydrocarbon on the calculations of substitution levels of organic layers with an omega-situated atomic tag ( $F$ in this case) is much smaller than the case of calculations based solely upon the $\mathrm{C} / \mathrm{Si}$ ratio. From the $\mathrm{C} / \mathrm{Si}$ ratio formula, the prefactor in front of the adventitious hydrocarbon thickness is $0.381 / \mathrm{nm}$, compared to $0.045 \mathrm{1} / \mathrm{nm}$ for the $\mathrm{F} / \mathrm{Si}$ formula. Therefore, if the adventitious hydrocarbon thickness is assumed to be anywhere between 0 and $1 \mathrm{~nm}$, the $\mathrm{C} / \mathrm{Si}$ formula predicts that the coverage is between the massive range of 15-53\% coverage compared to the F/Si formula, which predicts a range of 55-59\%, as shown in Figure 8.

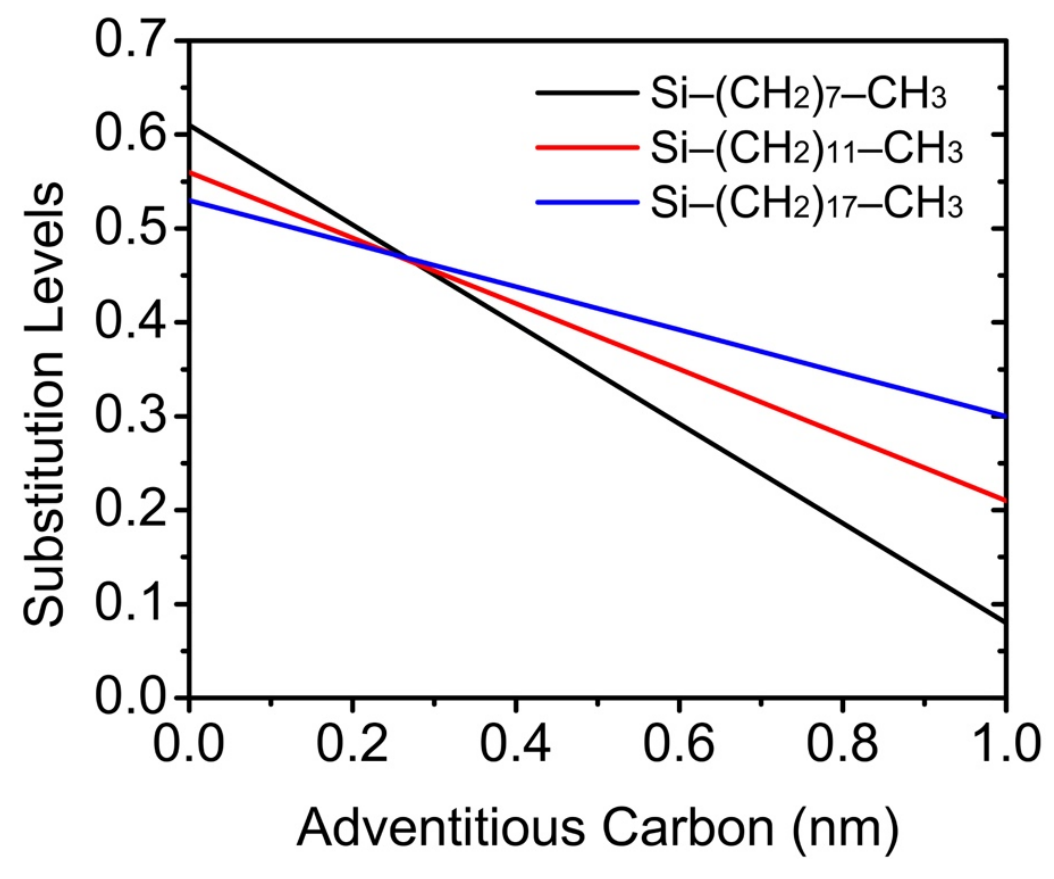


Figure 9. A scheme of the relationship between substitution levels and the thickness of adventitious hydrocarbon of $\equiv \mathrm{Si}(111)-\left(\mathrm{CH}_{2}\right)_{n}-\mathrm{CH}_{3}$-terminated surfaces (where $\mathrm{n}=7,11,17$ ), derived using the C/Si ratio from XPS data. The data from this figure are tabulated in Table 6.

For the three aliphatic $\equiv \mathrm{Si}-\left(\mathrm{CH}_{2}\right)_{n}-\mathrm{CH}_{3}$-terminated surfaces (where $\left.\mathrm{n}=7,11,17\right)$, the substitution levels all fall in the range of 53-61\%, assuming no adventitious carbon on the surface. From the three corresponding formulae for these interfaces derived from $\mathrm{C} / \mathrm{Si}$ ratios in Table 6 and Figure 9, we can see that the effect of adventitious hydrocarbon on calculated coverage decreases as the chain length increases from 8 carbons to 18 carbons. The presence of 1 $\mathrm{nm}$ adventitious hydrocarbon on the surface will lead to a calculated drop of substitution levels from $61 \%\left(d_{\mathrm{A}}=0\right)$ to $8 \%\left(d_{\mathrm{A}}=1 \mathrm{~nm}\right)$ for the $\equiv \mathrm{Si}-\left(\mathrm{CH}_{2}\right)_{7}-\mathrm{CH}_{3}$ surface, from $56 \%\left(d_{\mathrm{A}}=0\right)$ to $21 \%\left(d_{\mathrm{A}}=1 \mathrm{~nm}\right)$ for the $\equiv \mathrm{Si}-\left(\mathrm{CH}_{2}\right)_{11}-\mathrm{CH}_{3}$ surface, and from $53 \%\left(d_{\mathrm{A}}=0\right)$ to $27 \%\left(d_{\mathrm{A}}=1 \mathrm{~nm}\right)$ for the $\equiv \mathrm{Si}-\left(\mathrm{CH}_{2}\right)_{17}-\mathrm{CH}_{3}$ surface. Every Angstrom difference in adventitious hydrocarbon leads to a drop in calculated coverage of a $\mathrm{C}_{18}$-terminated surface by $2 \%$, while it changes that of a $\mathrm{C}_{8}$ layer by $-5 \%$. Because of the higher thickness and presumably more densely packed nature of the $\mathrm{C}_{18}$ monolayer compared to the $\mathrm{C}_{8}$ monolayer, the influence of the adventitious hydrocarbon on the longer $\mathrm{C}_{18}$-based layers is less with respect to the quantification of the substitution level. For the $\equiv \mathrm{Si}-\mathrm{S}-\left(\mathrm{CH}_{2}\right)_{17}-\mathrm{CH}_{3}$ surface on $\mathrm{Si}(111)$, with the buried sulfur bonded to the silicon surface, two formulae can be applied and compared, based on the $\mathrm{C} / \mathrm{Si}$ or $\mathrm{S} / \mathrm{Si}$ ratios, as shown in Table 6. The original experimental data for this surface functionalization chemistry was previously published in reference 43 . The formula using the $\mathrm{C} / \mathrm{Si}$ ratio, $\varphi=0.50-0.23 d_{\mathrm{A}}$ leads to a calculated substitution level of $\sim 50 \%$, assuming no adventitious carbon, but like the $\equiv \mathrm{Si}-\left(\mathrm{CH}_{2}\right)_{17}-\mathrm{CH}_{3}$-terminated surface, every Angstrom of adventitious hydrocarbon changes the substitution level of the $\equiv \mathrm{Si}-\mathrm{S}-\left(\mathrm{CH}_{2}\right)_{17}-\mathrm{CH}_{3}$ surface by $2 \%$. Using the $\mathrm{S} / \mathrm{Si}$ ratio, however, the calculated substitution level is independent of the thickness of adventitious hydrocarbon as it relies exclusively upon the $\mathrm{S} / \mathrm{Si}$ ratio, as shown in Eq 43. The substitution level calculated is $\sim 27 \%$. According to the formula in Table 6 that uses the C:Si ratio, $\varphi=0.50-0.23 d_{\mathrm{A}}, \sim 1 \mathrm{~nm}$ of adventitious carbon would result in a calculated coverage of $27 \%$. For all the calculations shown here, we assume that the packing density ratio of adventitious carbon to the alkyl chain is $0.8, \rho_{\mathrm{A}}$ $=0.8 \rho_{\mathrm{Au}}$ (vide supra), which will increase the estimated adventitious carbon thickness by $25 \%$; in any case, a small variation in $\rho_{\mathrm{A}}$ will lead to insignificant differences in the resulting calculations since the layer of adventitious carbon is already very thin, and is measured in terms of 
Angstroms. The relatively lower coverage of $\equiv \mathrm{Si}-\mathrm{S}-\left(\mathrm{CH}_{2}\right)_{17}-\mathrm{CH}_{3}$ compared to the aliphatic $\omega$ fluoro substituted $\equiv \mathrm{Si}-\left(\mathrm{CH}_{2}\right)_{11}-\mathrm{F}$ is almost certainly due to the larger surface-bound sulfur atoms that obstruct the neighbouring $\mathrm{Si}-\mathrm{H}$ groups and prevent close packing, leading to the overall lower substitution level. The lower substitution level of the $\equiv \mathrm{Si}-\mathrm{S}-\left(\mathrm{CH}_{2}\right)_{17}-\mathrm{CH}_{3}$ interface compared to the $\equiv \mathrm{Si}-\left(\mathrm{CH}_{2}\right)_{17}-\mathrm{CH}_{3}$ interface is also reflected in the slightly lower static water contact angle $\left(108^{\circ}\right.$ versus $109-112^{\circ}$, respectively, Table 2$)$, which points to a more disordered interface. A more disordered interface would be expected to have a higher surface energy and thus a higher propensity to getter adventitious carbon. ${ }^{78-80}$

The accuracy of every measurement of surface coverage is therefore limited by contamination. Here we show how adventitious physisorbed hydrocarbons could have a large influence on calculations of surface coverage that rely upon the ratio of silicon:carbon by XPS. Use of surface functionalizing agents with a heteroatom tag renders the calculations less susceptible to the effect of physisorbed hydrocarbons, but incomplete cleaning and/or rinsing of a surface after carrying out functionalization of a surface with an alkene with a fluorine tag may not remove all unreacted alkene, and would lead to higher ratios of F:Si if contaminated in this way. In a similar fashion, residual solvent from the reaction could also remain embedded within the formed monolayer, also skewing results. Strict attention to careful rinsing of the functionalized surfaces with the highest purity solvents, and completing the rinsing process with a volatile solvent like pentane or dichloromethane. Moreover, the use of a heteroatom allows for two independent measurements of the substitution level by using the XPS intensity ratios of both heteroatom:silicon and carbon:silicon. Given that both of these measurements must necessarily give the same answer, this approach provides an estimate of the thickness of the adventitious carbon and provides an internal consistency check of whether these derived numbers are physically reasonable.

The critical conclusion of this work is that the error in estimating the substitution level is an order of magnitude lower when using a heteroatomic tag, such as $\mathrm{S}$ or $\mathrm{F}$, compared to relying solely upon the ratio of carbon to silicon. When relying upon the $\mathrm{C} / \mathrm{Si}$ ratio, small differences in the thickness of adventitious carbon on the surface of a monolayer may result in a substantial difference of the calculated coverage. Our recommendation would be, when determining the level of substitution on a surface, would be to incorporate a small unobtrusive tag, such as a 
monofluorine substitution (as is the case with 11-fluoro-1-undecene) to use for the coverage determination, at least as a comparison. The results here are summarized in a flow chart, shown in Figure 10, that maps out the appropriate formula to use for a given functionalization on $\mathrm{Si}(111)$.

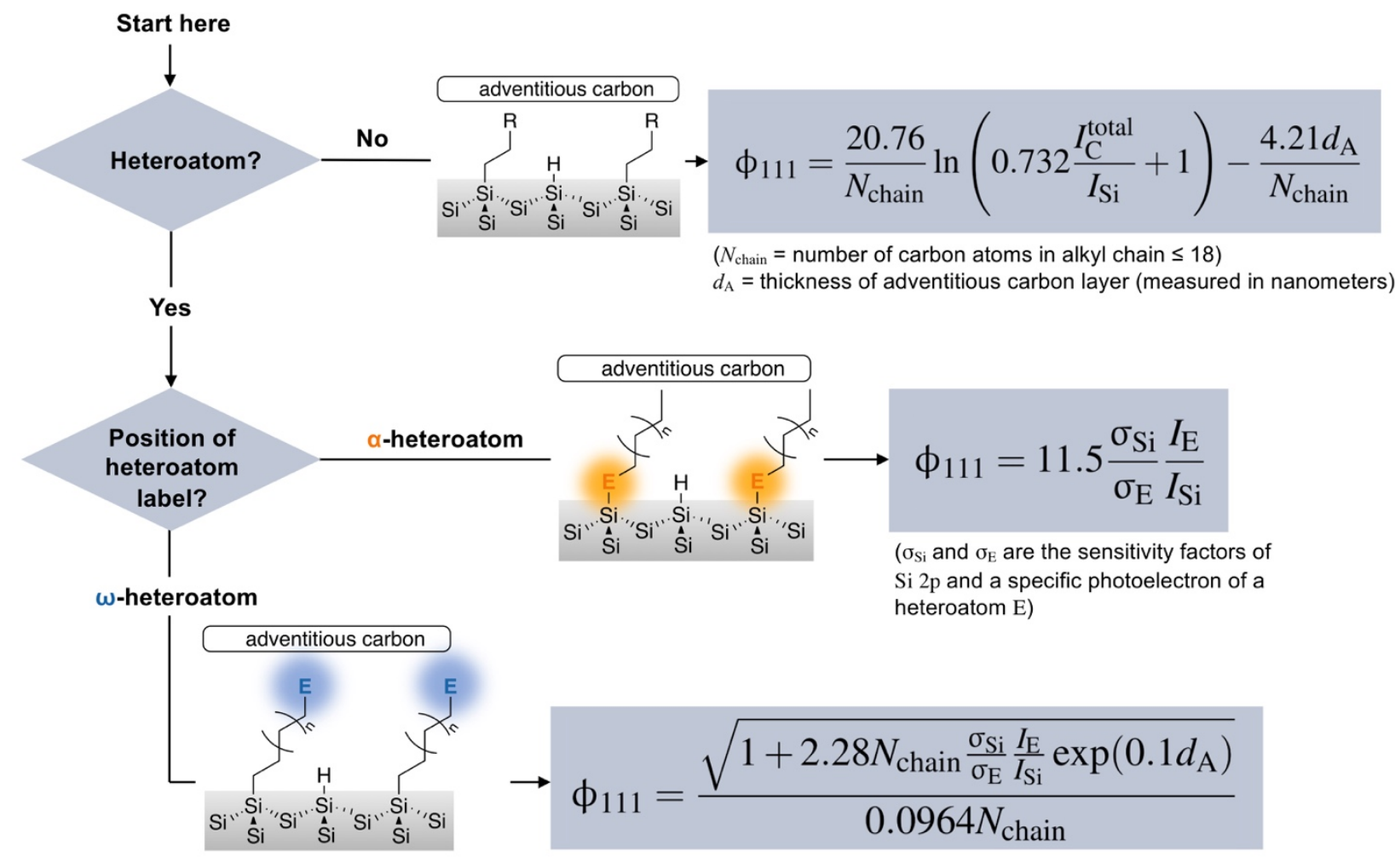

Functionalizing $\mathrm{Si}(\mathbf{1 0 0})$ and not $\mathrm{Si}(111) ? \longrightarrow \phi_{100}=1.15 \phi_{111}$

Figure 10. Algorithm that maps out the corresponding quantification formula for the substitution level of Si-H groups on silicon surface by pure alkyl monolayers, monolayers with $\omega$ heteroatom, and monolayer with $\alpha$-heteroatom. $I_{\mathrm{Si}}, I_{\mathrm{C}}{ }^{\text {total }}$, and $I_{\mathrm{E}}$, are the signal intensities of photoelectrons of Si 2p, C 1s, and a specific element, respectively, from XPS data. The generalized equations without the evaluation of constants can be found in Eqs. 23, 44 and 27 respectively. For all constants the electron attenuation lengths are evaluated using an XPS photon source with an energy of $1487 \mathrm{eV}$. 


\section{- Conclusions}

XPS is the most commonly used method to determine surface coverage of monolayers on $\mathrm{Si}(111)-\mathrm{H}$ surfaces, and typically relies upon the ratio of silicon to carbon. In this work, we went back into the roots of these commonly used calculations, and reconsidered the effects of substitution levels on critical values such as attenuation length, the effect of adventitious hydrocarbon contamination, and the utility of heteroatom tags. A new revised set of models and formulae for the quantification of substitution levels was developed. Inclusion of a heteroatom tag such as $\mathrm{F}$ and $\mathrm{S}$ will reduce the error in the quantification of the substitution level. While heteroatom tags may not be desirable in the final surface for a given application, they can be used as a model surface with which to compare analytical methods.

\section{ASSOCIATED CONTENT}

\section{AUTHOR INFORMATION}

\section{Corresponding Authors}

*Emails: eluber@ualberta.ca; jburiak@ualberta.ca

\section{ORCIDs}

Minjia Hu: 0000-0002-1140-9907

Erik J. Luber: 0000-0003-1623-0102

Jillian M. Buriak: 0000-0002-9567-4328

\section{Notes}

The authors declare no competing financial interest.

\section{Acknowledgements}

This work was supported by grants from the Natural Sciences and Engineering Research Council (NSERC, grant numbers RGPIN-2014-05195 and RGPIN 2019-04346), Alberta Innovates Technology Futures (AI graduate fellowships to M.H., and grant numbers CTDP-G2018000919 and AITF iCORE IC50-T1 G2013000198), Future Energy Systems of the University of Alberta (T12-P04), and the Canada Research Chairs Program (CRC 207142). 


\section{References}

(1) Veerbeek, J.; Huskens, J. Applications of Monolayer-Functionalized H-Terminated Silicon Surfaces: A Review. Small Methods 2017, 1, 1700072.

(2) Gao, F.; Teplyakov, A. V. Challenges and Opportunities in Chemical Functionalization of Semiconductor Surfaces. Appl. Surf. Sci. 2017, 399, 375-386.

(3) Gonçales, V. R.; Lian, J.; Gautam, S.; Tilley, R. D.; Gooding, J. J. Functionalized Silicon Electrodes in Electrochemistry. Annu. Rev. Anal. Chem. 2020, 13 (1), null. https://doi.org/10.1146/annurev-anchem-091619-092506.

(4) Rosenbluth, M. L.; Lieber, C. M.; Lewis, N. S. 630-mV Open Circuit Voltage, 12\% Efficient $n$-Si Liquid Junction. Appl. Phys. Lett. 1984, 45, 423-425.

(5) Bansal, A.; Lewis, N. S. Electrochemical Properties of (111)-Oriented $n$-Si Surfaces Derivatized with Covalently- Attached Alkyl Chains. J. Phys. Chem. B 1998, 102, 10671070 .

(6) Rosenbluth, M. L.; Lewis, N. S. Kinetic Studies of Carrier Transport and Recombination at the $n$-Silicon Methanol Interface. J. Am. Chem. Soc. 1986, 108, 4689-4695..

(7) Gouget-Laemmel, A. C.; Yang, J.; Lodhi, M. A.; Siriwardena, A.; Aureau, D.; Boukherroub, R.; Chazalviel, J.-N.; Ozanam, F.; Szunerits, S. Functionalization of Azide-Terminated Silicon Surfaces with Glycans Using Click Chemistry: XPS and FTIR Study. J. Phys. Chem. C 2013, 117, 368-375.

(8) Alderman, N.; Danos, L.; Grossel, M. C.; Markvart, T. Kelvin Probe Studies of Alkyl Monolayers on Silicon (111) for Surface Passivation. RSC Adv. 2013, 3, 20125-20131.

(9) Kronik, L.; Shapira, Y. Surface Photovoltage Phenomena: Theory, Experiment, and Applications. Surf. Sci. Rep. 1999, 37, 1-206.

(10) Fabre, B. Functionalization of Oxide-Free Silicon Surfaces with Redox-Active Assemblies. Chem. Rev. 2016, 116, 4808-4849.

(11) Veerbeek, J.; Firet, N. J.; Vijselaar, W.; Elbersen, R.; Gardeniers, H.; Huskens, J. Molecular Monolayers for Electrical Passivation and Functionalization of Silicon-Based Solar Energy Devices. ACS Appl. Mater. Interfaces 2017, 9, 413-421.

(12) Kashi, M. B.; Wu, Y.; Gonçales, V. R.; Choudhury, M. H.; Ciampi, S.; Gooding, J. J. SiliconSAM-AuNP Electrodes: Electrochemical "Switching" and Stability. Electrochem. Commun. 2016, 70, 28-32.

(13) Vogel, Y. B.; Molina, A.; Gonzalez, J.; Ciampi, S. Quantitative Analysis of Cyclic Voltammetry of Redox Monolayers Adsorbed on Semiconductors: Isolating Electrode Kinetics, Lateral Interactions, and Diode Currents. Anal. Chem. 2019, 91, 5929-5937. 
(14) Pekarek, R. T.; Celio, H.; Rose, M. J. Synthetic Insights into Surface Functionalization of Si(111)-R Photoelectrodes: Steric Control and Deprotection of Molecular Passivating Layers. Langmuir 2018, 34, 6328-6337.

(15) Ahmad, S. A. A.; Ciampi, S.; Parker, S. G.; Gonçales, V. R.; Gooding, J. J. Forming Ferrocenyl Self-Assembled Monolayers on $\mathrm{Si}(100)$ Electrodes with Different Alkyl Chain Lengths for Electron Transfer Studies. ChemElectroChem 2019, 6, 211-220.

(16) Mondal, P. C.; Fontanesi, C. Electrochemistry of Metalloproteins Attached through Functional Self-Assembled Monolayers on Gold and Ferromagnetic Electrodes. ChemPhysChem 2018, 19, 60-66.

(17) Gonçales, V. R.; Lian, J.; Gautam, S.; Hagness, D.; Yang, Y.; Tilley, R. D.; Ciampi, S.; Gooding, J. J. Heterojunctions Based on Amorphous Silicon: A Versatile Surface Engineering Strategy To Tune Peak Position of Redox Monolayers on Photoelectrodes. $J$. Phys. Chem. C 2020, 124, 836-844.

(18) Vilan, A.; Cahen, D. Chemical Modification of Semiconductor Surfaces for Molecular Electronics. Chem. Rev. 2017, 117, 4624-4666.

(19) Boucher, D. G.; Speller, J. R.; Han, R.; Osterloh, F. E.; Rose, M. J. Decoupling Effects of Surface Recombination and Barrier Height on P-Si(111) Photovoltage in Semiconductor|Liquid Junctions via Molecular Dipoles and Metal Oxides. ACS Appl. Energy Mater. 2019, 2, 66-79.

(20) King, L. A.; Hellstern, T. R.; Park, J.; Sinclair, R.; Jaramillo, T. F. Highly Stable Molybdenum Disulfide Protected Silicon Photocathodes for Photoelectrochemical Water Splitting. ACS Appl. Mater. Interfaces 2017, 9, 36792-36798.

(21) Zhu, Y.-L.; Yuan, J.-H.; Song, Y.-Q.; Wang, S.; Xue, K.-H.; Xu, M.; Cheng, X.-M.; Miao, X.-S. Two-Dimensional Silicon Chalcogenides with High Carrier Mobility for Photocatalytic Water Splitting. J. Mater. Sci. 2019, 54, 11485-11496.

(22) Lopinski, G. P.; Wayner, D. D. M. Molecular Monolayers on Silicon Surfaces. In Properties of Single Organic Molecules on Crystal Surfaces; World Scientific Publishing Co., 2006; pp 287-331.

(23) Ciampi, S.; Eggers, P. K.; Le Saux, G.; James, M.; Harper, J. B.; Gooding, J. J. Silicon (100) Electrodes Resistant to Oxidation in Aqueous Solutions: An Unexpected Benefit of Surface Acetylene Moieties. Langmuir 2009, 25, 2530-2539.

(24) Nguyen Minh, Q.; Pujari, S. P.; Wang, B.; Wang, Z.; Haick, H.; Zuilhof, H.; van Rijn, C. J. M. Fluorinated Alkyne-Derived Monolayers on Oxide-Free Silicon Nanowires via One-Step Hydrosilylation. Appl. Surf. Sci. 2016, 387, 1202-1210.

(25) Qin, G.; Santos, C.; Zhang, W.; Li, Y.; Kumar, A.; Erasquin, U. J.; Liu, K.; Muradov, P.; Trautner, B. W.; Cai, C. Biofunctionalization on Alkylated Silicon Substrate Surfaces via "Click" Chemistry. J. Am. Chem. Soc. 2010, 132, 16432-16441. 
(26) Strother, T.; Cai, W.; Zhao, X.; Hamers, R. J.; Smith, L. M. Synthesis and Characterization of DNA-Modified Silicon (111) Surfaces. J. Am. Chem. Soc. 2000, 122, 1205-1209.

(27) Liao, W.; Wei, F.; Qian, M. X.; Zhao, X. S. Characterization of Protein Immobilization on Alkyl Monolayer Modified Silicon(111) Surface. Sens. Actuators B Chem. 2004, 101, 361367.

(28) Satheesh, U.; Prakash, P.; Devaprakasam, D. Highly Sensitive and Reliable Octyltrichlorosilane Coated Silicon Sensors for Nitrogen Gas Flow Detection. Sens. Actuators Phys. 2019, 285, 190-199.

(29) Li, Q.; Lu, N.; Wang, L.; Fan, C. Advances in Nanowire Transistor-Based Biosensors. Small Methods 2018, 2, 1700263.

(30) Veerbeek, J.; Steen, R.; Vijselaar, W.; Rurup, W. F.; Korom, S.; Rozzi, A.; Corradini, R.; Segerink, L.; Huskens, J. Selective Functionalization with PNA of Silicon Nanowires on Silicon Oxide Substrates. Langmuir 2018, 34, 11395-11404.

(31) Mirsian, S.; Khodadadian, A.; Hedayati, M.; Manzour-ol-Ajdad, A.; Kalantarinejad, R.; Heitzinger, C. A New Method for Selective Functionalization of Silicon Nanowire Sensors and Bayesian Inversion for its Parameters. Biosens. Bioelectron. 2019, 142, 111527.

(32) Mandal, T.; Beck, M.; Kirsten, N.; Lindén, M.; Buske, C. Targeting Murine Leukemic Stem Cells by Antibody Functionalized Mesoporous Silica Nanoparticles. Sci. Rep. 2018, 8, 989.

(33) Cicero, R. L.; Linford, M. R.; Chidsey, C. E. D. Photoreactivity of Unsaturated Compounds with Hydrogen-Terminated Silicon(111). Langmuir 2000, 16, 5688-5695.

(34) Wallart, X.; Henry de Villeneuve, C.; Allongue, P. Truly Quantitative XPS Characterization of Organic Monolayers on Silicon: Study of Alkyl and Alkoxy Monolayers on H-Si(111). J. Am. Chem. Soc. 2005, 127, 7871-7878.

(35) Liu, Y.; Yamazaki, S.; Yamabe, S.; Nakato, Y. A Mild and Efficient Si (111) Surface Modification via Hydrosilylation of Activated Alkynes. J. Mater. Chem. 2005, 15, 49064913.

(36) Scheres, L.; Giesbers, M.; Zuilhof, H. Organic Monolayers onto Oxide-Free Silicon with Improved Surface Coverage: Alkynes versus Alkenes. Langmuir 2010, 26, 4790-4795.

(37) Rijksen, B.; Pujari, S. P.; Scheres, L.; van Rijn, C. J. M.; Baio, J. E.; Weidner, T.; Zuilhof, H. Hexadecadienyl Monolayers on Hydrogen-Terminated Si(111): Faster Monolayer Formation and Improved Surface Coverage Using the Enyne Moiety. Langmuir 2012, 28, 6577-6588.

(38) Yu, H.; Webb, L. J.; Ries, R. S.; Solares, S. D.; Goddard, W. A.; Heath, J. R.; Lewis, N. S. Low-Temperature STM Images of Methyl-Terminated Si(111) Surfaces. J. Phys. Chem. B 2005, 109, 671-674. 
(39) Yu, H.; Webb, L. J.; Heath, J. R.; Lewis, N. S. Scanning Tunneling Spectroscopy of Methyland Ethyl-Terminated Si(111) Surfaces. Appl. Phys. Lett. 2006, 88, 252111.

(40) Hurley, P. T.; Nemanick, E. J.; Brunschwig, B. S.; Lewis, N. S. Covalent Attachment of Acetylene and Methylacetylene Functionality to Si(111) Surfaces: Scaffolds for Organic Surface Functionalization While Retaining $\mathrm{Si}-\mathrm{C}$ Passivation of Si(111) Surface Sites. J. Am. Chem. Soc. 2006, 128, 9990-9991.

(41) Pujari, S. P.; van Andel, E.; Yaffe, O.; Cahen, D.; Weidner, T.; van Rijn, C. J. M.; Zuilhof, H. Mono-Fluorinated Alkyne-Derived SAMs on Oxide-Free Si(111) Surfaces: Preparation, Characterization and Tuning of the Si Workfunction. Langmuir 2013, 29, 570-580.

(42) Pujari, S. P.; Filippov, A. D.; Gangarapu, S.; Zuilhof, H. High-Density Modification of HTerminated Si(111) Surfaces Using Short-Chain Alkynes. Langmuir 2017, 33, 14599-14607.

(43) Hu, M.; Hauger, T. C.; Olsen, B. C.; Luber, E. J.; Buriak, J. M. UV-Initiated Si-S, Si-Se, and $\mathrm{Si}-\mathrm{Te}$ Bond Formation on Si(111): Coverage, Mechanism, and Electronics. J. Phys. Chem. C 2018, 122, 13803-13814.

(44) Linford, M. R.; Chidsey, C. E. D. Alkyl Monolayers Covalently Bonded to Silicon Surfaces. J. Am. Chem. Soc. 1993, 115, 12631-12632.

(45) Linford, M. R.; Fenter, P.; Eisenberger, P. M.; Chidsey, C. E. D. Alkyl Monolayers on Silicon Prepared from 1-Alkenes and Hydrogen-Terminated Silicon. J. Am. Chem. Soc. 1995, 117, $3145-3155$.

(46) Sieval, A. B.; van den Hout, B.; Zuilhof, H.; Sudhölter, E. J. R. Molecular Modeling of Covalently Attached Alkyl Monolayers on the Hydrogen-Terminated Si(111) Surface. Langmuir 2001, 17, 2172-2181.

(47) Zhang, L.; Wesley, K.; Jiang, S. Molecular Simulation Study of Alkyl Monolayers on Si(111). Langmuir 2001, 17, 6275-6281.

(48) Yuan, S.-L.; Cai, Z.-T.; Jiang, Y.-S. Molecular Simulation Study of Alkyl Monolayers on the Si(111) Surface. New J. Chem. 2003, 27, 626-633.

(49) Solares, S. D.; Yu, H.; Webb, L. J.; Lewis, N. S.; Heath, J. R.; Goddard, W. A. Chlorination-Methylation of the Hydrogen-Terminated Silicon(111) Surface Can Induce a Stacking Fault in the Presence of Etch Pits. J. Am. Chem. Soc. 2006, 128, 3850-3851.

(50) Wong, K. T.; Lewis, N. S. What a Difference a Bond Makes: The Structural, Chemical, and Physical Properties of Methyl-Terminated Si(111) Surfaces. Acc. Chem. Res. 2014, 47, 30373044.

(51) Plymale, N. T.; Dasog, M.; Brunschwig, B. S.; Lewis, N. S. A Mechanistic Study of the Oxidative Reaction of Hydrogen-Terminated Si(111) Surfaces with Liquid Methanol. J. Phys. Chem. C 2017, 121, 4270-4282.. 
(52) Sinha, S.; Mukherjee, M. A Study of Adventitious Contamination Layers on Technically Important Substrates by Photoemission and NEXAFS Spectroscopies. Vacuum 2018, 148, $48-53$.

(53) Greczynski, G.; Hultman, L. C 1s Peak of Adventitious Carbon Aligns to the Vacuum Level: Dire Consequences for Material's Bonding Assignment by Photoelectron Spectroscopy. ChemPhysChem 2017, 18, 1507-1512.

(54) Mangolini, F.; McClimon, J. B.; Rose, F.; Carpick, R. W. Accounting for Nanometer-Thick Adventitious Carbon Contamination in X-Ray Absorption Spectra of Carbon-Based Materials. Anal. Chem. 2014, 86, 12258-12265.

(55) Landoulsi, J.; Genet, M. J.; Fleith, S.; Touré, Y.; Liascukiene, I.; Méthivier, C.; Rouxhet, P. G. Organic Adlayer on Inorganic Materials: XPS Analysis Selectivity to Cope with Adventitious Contamination. Appl. Surf. Sci. 2016, 383, 71-83.

(56) Gleason-Rohrer, D. C.; Brunschwig, B. S.; Lewis, N. S. Measurement of the Band Bending and Surface Dipole at Chemically Functionalized Si(111)/Vacuum Interfaces. J. Phys. Chem. C 2013, 117, 18031-18042.

(57) Pekarek, R. T.; Celio, H.; Rose, M. J. Synthetic Insights into Surface Functionalization of Si(111)-R Photoelectrodes: Steric Control and Deprotection of Molecular Passivating Layers. Langmuir 2018, 34, 6328-6337.

(58) Sieval, A. B.; van den Hout, B.; Zuilhof, H.; Sudhölter, E. J. R. Molecular Modeling of Alkyl Monolayers on the Si(111) Surface. Langmuir 2000, 16, 2987-2990.

(59) Sieval, A. B.; Vleeming, V.; Zuilhof, H.; Sudhölter, E. J. R. An Improved Method for the Preparation of Organic Monolayers of 1-Alkenes on Hydrogen-Terminated Silicon Surfaces. Langmuir 1999, 15, 8288-8291.

(60) Sieval, A. B.; Demirel, A. L.; Nissink, J. W. M.; Linford, M. R.; van der Maas, J. H.; de Jeu, W. H.; Zuilhof, H.; Sudhölter, E. J. R. Highly Stable Si-C Linked Functionalized Monolayers on the Silicon (100) Surface. Langmuir 1998, 14, 1759-1768.

(61) Sun, Q.-Y.; de Smet, L. C. P. M.; van Lagen, B.; Giesbers, M.; Thüne, P. C.; van Engelenburg, J.; de Wolf, F. A.; Zuilhof, H.; Sudhölter, E. J. R. Covalently Attached Monolayers on Crystalline Hydrogen-Terminated Silicon: Extremely Mild Attachment by Visible Light. $J$. Am. Chem. Soc. 2005, 127, 2514-2523.

(62) Huang, Y.-S.; Chen, C.-H.; Chen, C.-H.; Hung, W.-H. Fabrication of Octadecyl and Octadecanethiolate Self-Assembled Monolayers on Oxide-Free Si(111) with a One-Cell Process. ACS Appl. Mater. Interfaces 2013, 5, 5771-5776.

(63) Huck, L. A.; Buriak, J. M. UV-Initiated Hydrosilylation on Hydrogen-Terminated Silicon (111): Rate Coefficient Increase of Two Orders of Magnitude in the Presence of Aromatic Electron Acceptors. Langmuir 2012, 28, 16285-16293. 
(64) Liu, Y.; Shen, L. From Langmuir Kinetics to First- and Second-Order Rate Equations for Adsorption. Langmuir 2008, 24, 11625-11630.

(65) Greczynski, G.; Hultman, L. Compromising Science by Ignorant Instrument CalibrationNeed to Revisit Half a Century of Published XPS Data. Angew. Chem. 2020, 132, 5034-5038.

(66) Gleason-Rohrer, D. C.; Brunschwig, B. S.; Lewis, N. S. Measurement of the Band Bending and Surface Dipole at Chemically Functionalized Si(111)/Vacuum Interfaces. J. Phys. Chem. C 2013, 117, 18031-18042.

(67) Nemanick, E. J.; Hurley, P. T.; Webb, L. J.; Knapp, D. W.; Michalak, D. J.; Brunschwig, B. S.; Lewis, N. S. Chemical and Electrical Passivation of Single-Crystal Silicon(100) Surfaces through a Two-Step Chlorination/Alkylation Process. J. Phys. Chem. B 2006, 110, 1477014778.

(68) Bruce, J. P.; Oliver, D. R.; Lewis, N. S.; Freund, M. S. Electrical Characteristics of the Junction between PEDOT:PSS and Thiophene-Functionalized Silicon Microwires. ACS Appl. Mater. Interfaces 2015, 7, 27160-27166.

(69) Alloway, D. M.; Hofmann, M.; Smith, D. L.; Gruhn, N. E.; Graham, A. L.; Colorado, R.; Wysocki, V. H.; Lee, T. R.; Lee, P. A.; Armstrong, N. R. Interface Dipoles Arising from SelfAssembled Monolayers on Gold: UV-Photoemission Studies of Alkanethiols and Partially Fluorinated Alkanethiols. J. Phys. Chem. B 2003, 107, 11690-11699.

(70) Miura, Y. F.; Takenaga, M.; Koini, T.; Graupe, M.; Garg, N.; Graham, Robert L.; Lee, T. R. Wettabilities of Self-Assembled Monolayers Generated from CF3-Terminated Alkanethiols on Gold. Langmuir 1998, 14, 5821-5825.

(71) Lee, T. R.; Carey, R. I.; Biebuyck, H. A.; Whitesides, G. M. The Wetting of Monolayer Films Exposing Ionizable Acids and Bases. Langmuir 1994, 10, 741-749.

(72) Ulman, A.; Eilers, J. E.; Tillman, N. Packing and Molecular Orientation of Alkanethiol Monolayers on Gold Surfaces. Langmuir 1989, 5, 1147-1152.

(73) Bain, C. D.; Whitesides, G. M. Formation of Monolayers by the Coadsorption of Thiols on Gold: Variation in the Length of the Alkyl Chain. J. Am. Chem. Soc. 1989, 111, 7164-7175.

(74) Bain, C. D.; Whitesides, G. M. Attenuation Lengths of Photoelectrons in Hydrocarbon Films. J. Phys. Chem. 1989, 93, 1670-1673.

(75) Laibinis, P. E.; Bain, C. D.; Whitesides, G. M. Attenuation of Photoelectrons in Monolayers of N-Alkanethiols Adsorbed on Copper, Silver, and Gold. J. Phys. Chem. 1991, 95, 70177021.

(76) Park, J.-S.; Vo, A. N.; Barriet, D.; Shon, Y.-S.; Lee, T. R. Systematic Control of the Packing Density of Self-Assembled Monolayers Using Bidentate and Tridentate Chelating Alkanethiols. Langmuir 2005, 21, 2902-2911. 
(77) Tanuma, S.; Powell, C. J.; Penn, D. R. Calculations of Electron Inelastic Mean Free Paths. V. Data for 14 Organic Compounds over the 50-2000 EV Range. Surf. Interface Anal. 1994, 21, $165-176$.

(78) Ford, K.; Battersby, B. J.; Wood, B. J.; Gentle, I. R. The Production and Verification of Pristine Semi-Fluorinated Thiol Monolayers on Gold. J. Colloid Interface Sci. 2012, 370, $162-169$.

(79) Chaudhury, M. K.; Whitesides, G. M. Correlation Between Surface Free Energy and Surface Constitution. Science 1992, 255, 1230-1232.

(80) Kohli, R.; Mittal, K. L. Developments in Surface Contamination and Cleaning, Vol. 1: Fundamentals and Applied Aspects; William Andrew, 2015. 